\title{
A Generic Multi-Compartmental CNS Distribution Model Structure for 9 Drugs Allows Prediction of Human Brain Target Site Concentrations
}

\author{
Yumi Yamamoto' • Pyry A. Välitalo' • Dirk-Jan van den Berg ' Robin Hartman ' • \\ Willem van den Brink ${ }^{\prime}$ ' Yin Cheong Wong ${ }^{\prime}$ Dymphy R. Huntjens ${ }^{2} \cdot$ Johannes H. Proost ${ }^{3}$ • \\ An Vermeulen ${ }^{2}$. Walter Krauwinkel ${ }^{4}$. Suruchi Bakshi ${ }^{1}$ - Vincent Aranzana-Climent ${ }^{5}$. \\ Sandrine Marchand ${ }^{5}$. Claire Dahyot-Fizelier ${ }^{6} \cdot{\text { William } \text { Couet }^{5} \cdot \text { Meindert Danhof }}^{\prime}$ • \\ Johan G. C. van Hasselt ${ }^{\prime}$ • Elizabeth C. M. de Lange ${ }^{1,7}$
}

Received: 24 August 2016 / Accepted: 7 November 2016 / Published online: 18 November 2016

(C) The Author(s) 2016. This article is published with open access at Springerlink.com

\begin{abstract}
Purpose Predicting target site drug concentration in the brain is of key importance for the successful development of drugs acting on the central nervous system. We propose a generic mathematical model to describe the pharmacokinetics in brain compartments, and apply this model to predict human brain disposition.
\end{abstract}

Methods A mathematical model consisting of several physiological brain compartments in the rat was developed using rich concentration-time profiles from nine structurally diverse drugs in plasma, brain extracellular fluid, and two cerebrospinal fluid compartments. The effect of active drug transporters

Electronic supplementary material The online version of this article (doi: | 0. I 007/s | | 095-0 | 6-2065-3) contains supplementary material, which is available to authorized users.

Elizabeth C. M. de Lange

ecmdelange@lacdr.leidenuniv.nl

Division of Pharmacology, Cluster Systems Pharmacology, Leiden Academic Centre for Drug Research, Leiden University,

Leiden, The Netherlands

2 Quantitative Sciences, Janssen Research \& Development, a Division of Janssen Pharmaceutica NV, Beerse, Belgium

3 Division of Pharmacokinetics, Toxicology and Targeting, University of Groningen, Groningen, The Netherlands

4 Department of Clinical Pharmacology \& Exploratory Development, Astellas Pharma BV, Leiden, The Netherland

5 Department of Medicine and Pharmacy, University of Poitiers, Poitiers, France

6 Department of Anaesthesiology and Intensive Care Medicine, University Hospital Center of Poitiers, Poitiers, France

7 Leiden University Gorlaeus Laboratories, Einsteinweg 55, 2333CC Leiden, The Netherlands was also accounted for. Subsequently, the model was translated to predict human concentration-time profiles for acetaminophen and morphine, by scaling or replacing system- and drug-specific parameters in the model.

Results A common model structure was identified that adequately described the rat pharmacokinetic profiles for each of the nine drugs across brain compartments, with good precision of structural model parameters (relative standard error $<37.5 \%$ ). The model predicted the human concentrationtime profiles in different brain compartments well (symmetric mean absolute percentage error $<90 \%$ ).

Conclusions A multi-compartmental brain pharmacokinetic model was developed and its structure could adequately describe data across nine different drugs. The model could be successfully translated to predict human brain concentrations.

KEY WORDS blood-brain barrier · central nervous system (CNS) · human prediction · pharmacokinetics · translational model

$\begin{array}{ll}\text { ABBREVIATIONS } \\ \text { BBB } & \text { Blood-brain barrier } \\ \text { BCSFB } & \text { Blood-cerebrospinal fluid barrier } \\ \text { brain }_{E C F} & \text { Brain extracellular fluid compartment } \\ \text { brain }_{I C F} & \text { Brain intracellular fluid compartment } \\ \text { CNS } & \text { Central nervous system } \\ \text { CSF } & \text { Cerebrospinal fluid } \\ \text { CSFCM } & \text { Compartment of cerebrospinal fluid in cisterna } \\ \text { CSFEV }_{E} & \begin{array}{l}\text { Compagna } \\ \text { external-ventricular drainage }\end{array} \\ \text { CSF }_{L V} & \text { Compartment of cerebrospinal fluid in lateral } \\ & \text { ventricle }\end{array}$

\section{ABBREVIATIONS}

BCSFB Blood-cerebrospinal fluid barrier

brain $_{\mathrm{ECF}} \quad$ Brain extracellular fluid compartment

Brain intracellular fluid compartment

Central nervous system

Cerebrospinal fluid

CSF $_{\mathrm{CM}}$ Compartment of cerebrospinal fluid in cisterna magna external-ventricular drainage ventricle 


\begin{tabular}{|c|c|}
\hline $\mathrm{CSF}_{\mathrm{SAS}}$ & $\begin{array}{l}\text { Compartment of cerebrospinal fluid in subarach- } \\
\text { noid space }\end{array}$ \\
\hline $\mathrm{CSF}_{\mathrm{TFV}}$ & $\begin{array}{l}\text { Compartment of cerebrospinal fluid in third and } \\
\text { fourth ventricle }\end{array}$ \\
\hline ECF & Extracellular fluid \\
\hline EVD & External-ventricular drainage \\
\hline ICF & Intracellular fluid \\
\hline IPRED & Individual prediction \\
\hline PF & Perfusion fluid \\
\hline PK & Pharmacokinetic \\
\hline PRED & Population predictions \\
\hline$S A_{B B B}$ & Surface area of blood-brain barrier \\
\hline$S A_{B C S F S B}$ & Surface area of blood-cerebrospinal barrier \\
\hline SMAPE & Symmetric mean absolute percentage error \\
\hline SPE & Solid phase extraction \\
\hline TBI & Traumatic brain injury \\
\hline
\end{tabular}

\section{INTRODUCTION}

Central nervous system (CNS) drug development suffers from $91 \%$ attrition rate and especially the success rate in phase II is very low $(1,2)$. The primary reasons for attrition are safety issues (3). Although the underlying physiological and pharmacological reasons for such failures are often not fully known they are likely related to a lack of knowledge or failure to account for a combination of on- and off-target site concentrations, target interaction and downstream signal processing. The first step in this cascade, obtaining quantitative insight into CNS target site concentration kinetics, is already a major challenge, and has been suggested as a major factor contributing to failure of novel drug candidates (4). During clinical drug development, typically only drug plasma concentrations are considered as marker for drug exposure, because quantifying drug concentrations in the brain is challenging. Hence, the ability to predict brain concentrations based on plasma data is highly relevant to further optimize CNS drug development.

The prediction of brain target site concentrations is controlled by several factors. First, the poorly penetrable bloodbrain barrier (BBB) and the blood-cerebrospinal barrier (BCSFB) (5) limit passage of drugs from the systemic circulation into the brain. These barriers are associated with limited passive diffusion, and in addition various active transport and drug metabolism processes that systematically administered drugs need to pass. Second, the brain can be further subdivided into several distinct physiological compartments, including the brain extracellular fluid (ECF), brain intracellular fluid (ICF), and multiple cerebrospinal fluid (CSF) compartments. The specific disposition characteristics across these specific compartments further determines drug target site concentrations. Third, CNS drug target site concentrations are mediated by physiological flows including the microvascular blood flow, and brain ECF and CSF flows. Lastly, drug protein binding and the localized $\mathrm{pH}$ in specific subcompartments further affect ultimate brain target site concentrations.

Passive drug transport processes are mediated through a combination of drug permeability properties, transmembrane transport routes, and the surface areas of the $\mathrm{BBB}\left(\mathrm{SA}_{\mathrm{BBB}}\right)$ and $\mathrm{BCSFB}\left(\mathrm{SA}_{\mathrm{BCSFB}}\right)(5)$. Active drug transport is mediated by transport proteins such as $\mathrm{P}$-glycoprotein $(\mathrm{P}$ gp), multidrug resistance-associated protein (MRPs), organic anion transporters $(\mathrm{OATs})$, and organic anion transporting polypeptides (OATPs). Even though the function and localization of these transporters has been extensively investigated in in-vitro and in-vivo studies, their precise functions is in some cases not fully understood (6).

Several experimental preclinical models have been developed to assess drug distribution to brain compartments. These models differ in terms of temporal and spatial resolution, and in their consideration of drug protein binding (7-10). For example, the combinatorial mapping approach has been recently introduced using unbound drug concentration with the brain slice technique $(10,11)$. This approach can predict unbound drug CNS exposure at steady state in multiple brain compartments, but does not allow temporal characterization of drug concentration changes. Positron emission tomography (PET) is sometimes used also clinically, as a non-invasive imaging method to visualize spatiotemporal drug distribution in the brain. However, PET scan signals cannot distinguish parent compounds from their metabolites, or bound and unbound drug compounds in the brain (12). Finally, microdialysis allows serial sampling in multiple physiological compartments of unbound drug concentrations, hence is suited to characterize the time profile of drug concentrations in the brain (13).

In order to capture the time profile and complexity of interacting factors governing drug distribution across brain compartments as determined by microdialysis methods, mathematical modeling represents an indispensable tool. Specifically, physiologically based pharmacokinetic (PBPK) models are of interest, as these models aim to distinguish between system- and drug-specific parameters, allowing for translational predictions by scaling or replacing system- or drug-specific parameters from the rat to man (14). Several (semi-) PBPK models for CNS drug distribution have been published, with different levels of complexity (15-20). However, these models did not yet include validations of predicted human CNS concentrations (21). Recently, Gaohoa et al published a CNS PBPK model, which consists of four compartments such as brain blood volume, brain mass, cranial CSF and spinal CSF. This model was validated with human acetaminophen and phenytoin data. However, a limitation of this model is the lack of consideration of a brain extracellular

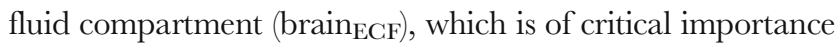


for prediction of receptor binding kinetics for drugs acting on membrane bound receptors and ultimately drug efficacy (22).

Previously we have developed separate semi-physiological CNS PBPK models for three drugs based on microdialysis experiments in rats, which included unbound drug concentration-time profiles across multiple brain compartments (23-25). These models described the data well, but resulted in different individual model structures for each of these drugs.

The purpose of the current work was to develop a more generally applicable model structure that can be used to predict drug target site concentration-time profiles in human brain compartments based on rat pharmacokinetic (PK) studies. To this aim, we used published and newly generated datasets for a larger number of drugs, and we performed rigorous model validation on external datasets. Furthermore, the impact of key drug transporters was also included in our model. Finally, we investigated the performance of the developed model structure to predict human brain concentration-time profiles for acetaminophen and morphine.

\section{MATERIALS AND METHODS}

\section{Data for Model Development}

An overview of experimental data for nine compounds with different physicochemical characteristics used for model development is provided in Table I. The physicochemical characteristics of the nine compounds are provided in Table SI. Data on 6 compounds were previously published, as indicated in Table I. For three compounds (paliperidone, phenytoin and risperidone), data were newly produced after single intravenous administration, as described below.

For some of the drugs, active transport inhibitors were coadministered intravenously to characterize the effect of P-gp, MRP, OATs and OATPs, as indicated in Table I. The transport inhibitors included were probenecid as an inhibitor of MRPs, OATs and OATPs, and GF120918 or tariquidar as inhibitor of P-gp.

\section{Data for External Model Validation}

For an external validation of the model, we used two separate rat datasets for acetaminophen and remoxipride, as indicated in Table I. The acetaminophen data was previously published, the remoxipride data was newly generated as described in the experimental section. For acetaminophen and remoxipride, two separate experimental datasets were available. For each drug, one of these datasets was used for model development, whilst the second dataset was used for external validation. The external validation with these second sets of data allows assessment of the robustness of our model predictions with respect to a different experiment and variation in experimental design.

\section{Animals}

Animal study protocols were approved by the Animal Ethics Committee of Leiden University and all animal experiments were performed in accordance with the Dutch Law of Animal Experimentation (for approval numbers see Table SII). Male Wistar rats (225-275 g, Charles River, The Netherlands) were housed in groups for a few days (513 days) under standard environmental conditions with ad libitum access to food (Laboratory chow, Hope Farms, Woerden, The Netherlands) and acidified water. Between surgery and experiments, the animals were kept individually in Makrolon type three cages for 7 days to recover from surgical procedures.

\section{Surgery}

Rats were anesthetized ( $5 \%$ isoflurane for induction, $1-2 \%$ as maintenance), and subsequently received cannulas in the femoral artery for serial blood sampling, and in the femoral vein for drug administration, respectively. Subsequently, microdialysis guides were inserted into different brain locations. The animals were allowed to recover for 1 week before the experiments were performed. One day before the experiment, the microdialysis dummies were replaced by microdialysis probes. For details on guides, probes and locations see Table SII.

\section{Microdialysis and Drug Administration}

Experiments generally started at 9:00 a.m. to minimize the influence of circadian rhythms. Microdialysis probes were continuously flushed with microdialysis perfusion fluid $(\mathrm{PF})$ until equilibration before the start of drug administration. Drugs were administered at $t=0 \mathrm{~h}$ by intravenous infusion through the cannula implanted in the femoral vein. For the quantification of active drug transport, the active transport inhibitor was administered before the drug's administration. The general procedure of microdialysis is depicted in Fig. 1. Dosage and infusion time for each drug and the active transport inhibitor were summarized in Table I, and the composition of microdialysis $\mathrm{PF}$ and flow rate of microdialysis $\mathrm{PF}$ are summarized in Table SII.

\section{Bioanalytical methods}

The developed analytical methods for risperidone, paliperidone, phenytoin and remoxipride are described below. 


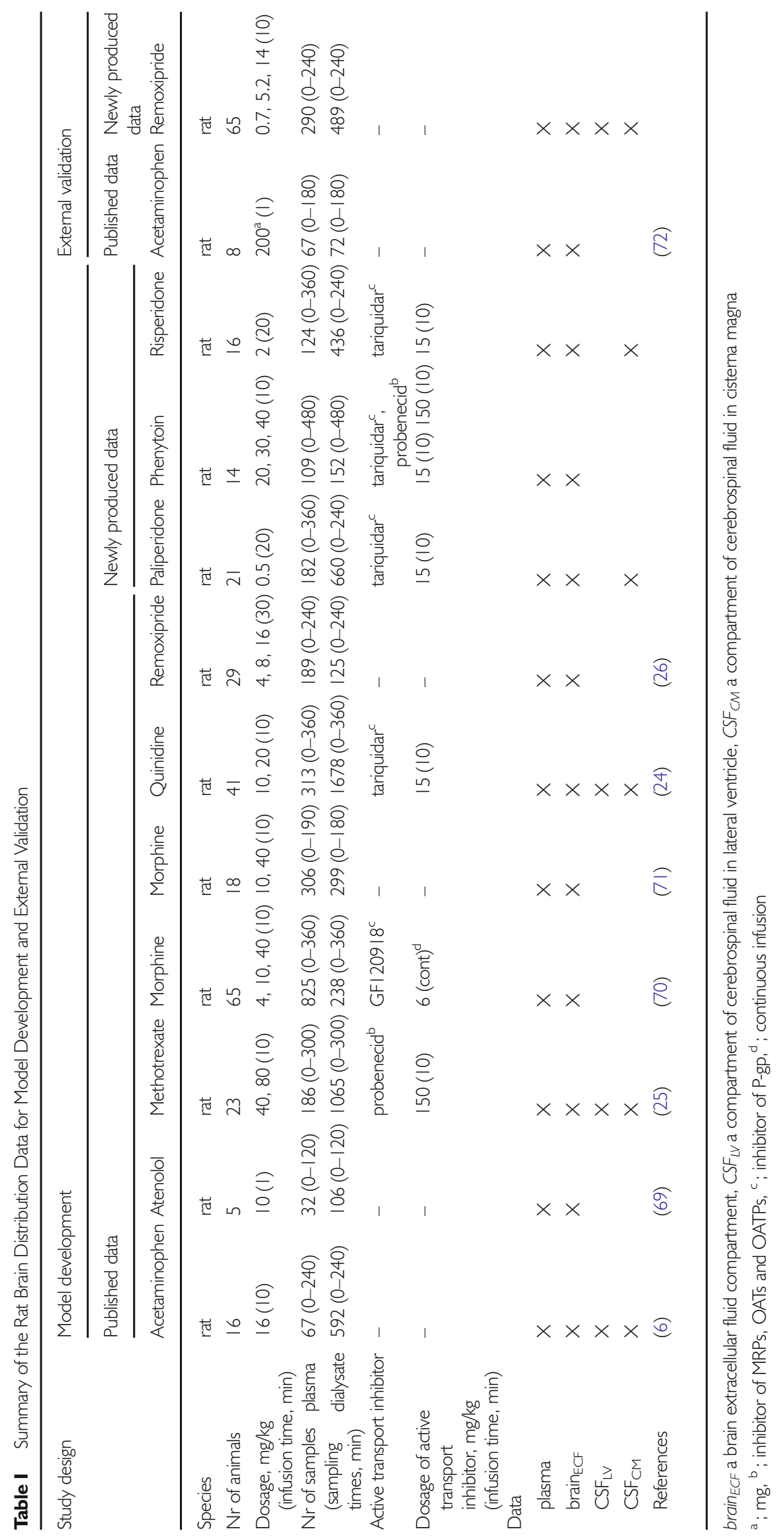


Fig. I Microdialysis procedures for the compounds used for the development of the multicompartmental brain PK model.

No active transport inhibitor

9 compounds

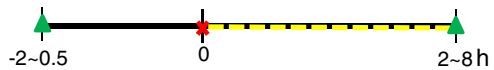

Single active transport inhibitor

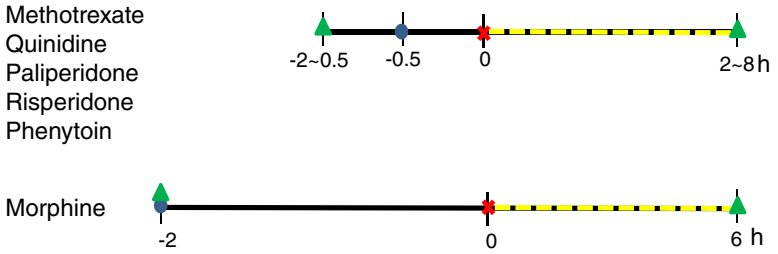

Double active transport inhibitor

Phenytoin

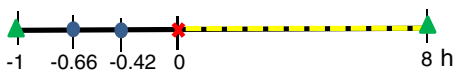

$\Delta$ Start/end of the microdialysis

* Administration of the compounds

- Administration of the active transport inhibitors

\section{Chemicals and Reagents}

For all procedures, nanopure lab water $(18.2 \mathrm{M} \Omega \mathrm{cm})$ was used. All chemicals used were obtained from Sigma Aldrich (Zwijndrecht, The Netherlands), and analytical grade unless stated otherwise. The internal standards risperidone-D4 and paliperidone-D4 were purchased from Toronto Research Chemicals (Toronto, Ontario, Canada). Remoxipride. HCl was obtained from TOCRIS (Bristol, United Kingdom). Tariquidar (TQD, XR9576) was obtained from Xenova group PLC (Cambridge, United Kingdom). Ammonium formate, ammonium bicarbonate (ULC/MS grade), acetonitrile (LG-MS grade), methanol, isopropanol, and formic acid (ULC/MS grade) were obtained from Biosolve B.V. (Valkenswaard, The Netherlands). Sodium hydroxide was obtained from Baker (Deventer, The Netherlands).

\section{Sample Preparation of Plasma}

\section{Risperidone and Paliperidone}

The calibration curve was in a range of 5 to $1000 \mathrm{ng} / \mathrm{ml}$. Quality controls (QC's) were prepared in blank rat plasma at three different concentration levels and stored at $-20^{\circ} \mathrm{C}$. The lower limit of quantification (LLOQ) for both risperidone and paliperidone was $5 \mathrm{ng} / \mathrm{ml}$. To $20 \mu \mathrm{l}$ of plasma, $20 \mu \mathrm{l}$ of internal standard solution (risperidone-D4 and paliperidoneD4) and $20 \mu \mathrm{l}$ water (or $20 \mu \mathrm{l}$ calibration solution in the case of the calibration curve) were added. After brief vortexing, $1 \mathrm{ml}$ of acetonitrile was added. Brief vortexing and subsequent centrifugation at 10,000 $\mathrm{g}$ led to a clear supernatant, which was transferred to a glass tube and evaporated in the vortex evaporator (Labconco, Beun de Ronde, Breda, The Netherlands). The residue was redissolved in $200 \mu \mathrm{l}$ of $2 \%$ methanol, $10 \mathrm{mM}$ ammonium formate, $\mathrm{pH} 4.1$ and processed in according to the solid phase extraction (SPE)- liquid chromatography (LG) method.

\section{Phenytoin}

$20 \mu \mathrm{l}$ of plasma sample was mixed with $20 \mu \mathrm{l}$ of water in an Eppendorf vial. An aliquot of $40 \mu \mathrm{l}$ acetonitrile was added for protein precipitation. After centrifugation at $11,000 \mathrm{~g}$ for $10 \mathrm{~min}, 40 \mu \mathrm{l}$ of supernatant was mixed with $40 \mu \mathrm{l}$ ammonium acetate buffer ( $\mathrm{pH}$ 5.0). Calibration was performed by adding $20 \mu \mathrm{l}$ of calibration solution to $20 \mu \mathrm{l}$ of blank plasma, using the same clean-up procedure. The calibration solutions ranged from 0.2 to $100 \mu \mathrm{g} / \mathrm{ml} .30 \mu \mathrm{l}$ was injected into the highperformance liquid chromatography (HPLC) system. The LLOQ was $250 \mathrm{ng} / \mathrm{ml}$.

\section{Remoxipride}

Sample preparation was performed according to Stevens et al (26). Briefly, $20 \mu \mathrm{l}$ of sample was mixed with $20 \mu \mathrm{l}$ of water and $20 \mu \mathrm{l}$ internal standard (raclopride). Proteins were precipitated with $6 \%$ perchloric acid and centrifugation. After addition of sodium carbonate, $10 \mu \mathrm{l}$ was injected into the SPE-LC system.

\section{Sample Preparation for Microdialysates}

\section{Risperidone and Paliperidone}

The calibration curve for the microdialysis samples was prepared in buffered PF (composition in Table SII). The concentrations were in the range of 0.1 to $20 \mathrm{ng} / \mathrm{ml}$. QC's were prepared using a different batch of buffered PF. Before injection of $10 \mu \mathrm{l}$ into the LG system, the microdialysate samples were diluted with internal standard solution in a ratio of $1: 1 \mathrm{v} /$ v. The internal standard solution consisted of $100 \mathrm{ng} / \mathrm{ml}$ 
risperidone-D4 and paliperidone-D4 in nanopure water. The LLOQs for risperidone and paliperidone were 0.4 and $0.2 \mathrm{ng} / \mathrm{ml}$, respectively.

\section{Phenytoin}

Calibration curves were made in minimal PF at a concentration range of 25 to $5000 \mathrm{ng} / \mathrm{ml}$. QC's were prepared using a different batch of buffered PF. Of a typical sample that consisted of $40 \mu \mathrm{l}$ of microdialysate, $30 \mu \mathrm{l}$ was injected into the HPLC system. The LLOQ was $25 \mathrm{ng} / \mathrm{ml}$.

\section{Remoxipride}

Calibration curves were prepared in buffered PF. The calibration range was from 1 to $200 \mathrm{ng} / \mathrm{ml}$. QC's were prepared using a different batch of buffered PF. Samples were mixed in a $1: 1 \mathrm{v} / \mathrm{v}$ ratio with the internal standard raclopride $(100 \mathrm{ng} / \mathrm{ml})$ before injection of $5 \mu \mathrm{l}$ into the $\mathrm{LC}$ system. The LLOQ was $0.5 \mathrm{ng} / \mathrm{ml}$.

\section{Chromatography}

\section{Paliperidone and Risperidone}

SPE-LC Method. For plasma samples the SPE-method was applied. The SPE system consisted of a Hyphere C8 HD, SE column $(10 \times 2 \mathrm{~mm})$ (Spark Holland, Emmen, The Netherlands) in a cartridge holder and served for the clean-up of the sample. The cartridge holder was connected to a Gynkotek gradient pump (Thermo Scientific, Breda, The Netherlands) and a Waters 717 autosampler (Waters, Etten-Leur, The Netherlands). The MS Surveyor pump from Thermo Scientific (Breda, The Netherlands) provided the flow for the LC column, which was the same type as in the LC-method. The sample was injected onto the SPE, which was preconditioned with $2 \%$ methanol $(\mathrm{pH} 4.1)$. After 1 min of flushing, the SPE was switched into the LC system. After 4 min, the SPE was cleaned with 98\% methanol ( $\mathrm{pH} 4.1$ ) for $2 \mathrm{~min}$ and reconditioned with $2 \%$ methanol $(\mathrm{pH} 4.1)$. The flow of the SPE pump was $0.75 \mathrm{ml} / \mathrm{min}$. The flow of the LC system was $0.25 \mathrm{ml} / \mathrm{min}$. The gradient was from 10 to $90 \%$ methanol (1-8.5 min after injection). The SPE column was used for a maximum of 240 injections.

LC-Method. For microdialysates, LC-Method was applied. The separation of the active compounds was possible using

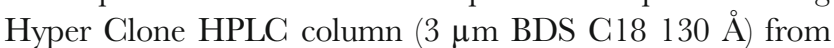
Phenomenex (Utrecht, The Netherlands) placed at $40^{\circ} \mathrm{C}$. The LC system was used at a flow of $0.25 \mathrm{ml} / \mathrm{min}$ using a linear gradient from 20 to $74 \%$ methanol (1-6 min after injection). Before the next injection, the column was re-equilibrated with $20 \%$ methanol for 2 min.

\section{Phenytoin}

HPLC Method and Detection. For both plasma and microdialysates samples an HPLC method was used. The mobile phase consisted of $15 \mathrm{mM}$ ammonium acetate adjusted to $\mathrm{pH} 5.0$ with acetic acid and acetonitrile in a 2:1 ratio (v/v). Separation was achieved using an Altima HP C18Amide HPLC column $(5 \mu \mathrm{m}, 150 \times 4.6 \mathrm{~mm})$ from Grace Alltech (Breda, The Netherlands). The injector was from Waters (Etten-Leur, The Netherlands). The LC pump (LC10 ADVP) was obtained from Shimadzu ('s-Hertogenbosch, The Netherlands). The ultraviolet (UV) detector (Spectroflow 757) was obtained from Applied Biosystems (Waltham, Massachusetts) and was used at a wavelength of $210 \mathrm{~nm}$. Data acquisition was achieved using Empower software from Waters (Etten-Leur, The Netherlands).

\section{Remoxipride}

SPE-LC Method. For the precipitated plasma samples, on-line SPE was combined with HPLC and mass spectrometry according to Stevens et al (26). Briefly, a pretreated sample was loaded into a Hysphere GP resin cartridge column $(10 \times 2 \mathrm{~mm})$ from Spark Holland (Emmen, The Netherlands) at pH 8.3 and flushed for 1 min. Elution was performed using a low $\mathrm{pH}$ and an Altima HP C18 column $(150 \times 1.0 \mathrm{~mm}, 5 \mu \mathrm{m})$.

LC-Method. For microdialysates, a Kinetex $2.6 \mu \mathrm{m}$ column $(50 \times 2.0 \mathrm{~mm}, \mathrm{XB}-\mathrm{C}-18)$ from Phenomenex (Utrecht, The Netherlands) was used at a flow of $0.6 \mathrm{ml} / \mathrm{min}$ and placed at $40{ }^{\circ} \mathrm{C}$. The system was a Nexera- $X 2$ UHPLC system, consisting of two ultra high performance liquid chromatography (UHPLG) pumps delivering the high pressure gradient. A SIL-30 AC auto sampler was used to inject $5 \mu \mathrm{l}$ of the microdialysis sample. The flow was diverted for the first $0.5 \mathrm{~min}$, while a gradient from 10 to $90 \%$ methanol in 1.5 min served to elute both remoxipride and raclopride to the mass spectrometer.

\section{Mass Spectrometry}

For risperidone, paliperidone and remoxipride, mass spectrometry was used to measure the concentrations. The mass spectrometer was a TSQ Quantum Ultra from Thermo Fisher Scientific (Breda, the Netherlands) and was used in MS/MS mode. Electrospray was used for ionization in the positive mode, nitrogen served as the desolvation gas and argon was used as collision gas. Data acquisition for both remoxipride and risperidone and paliperidone was performed using LCQuan 2.5 software from Thermo Scientific (Breda, The Netherlands). 
Risperidone and paliperidone had the following transitions $(\mathrm{m} / \mathrm{z}): 411.2 \rightarrow 191.1$ (risperidone), $415.2 \rightarrow 195.1$ (paliperidone), $415.2 \rightarrow 195.1$ (risperidone-D4), 431.2 $\rightarrow 211.1$ (paliperidone-D4). The scan width was set at $0.2 \mathrm{~m} / \mathrm{z}$, the scan time was $0.05 \mathrm{~s}$. Collision was performed at fixed voltages between 27 and $38 \mathrm{~V}$, using a skimmer offset of $2 \mathrm{~V}$.

The transitions $(\mathrm{m} / \mathrm{z})$ were $371 \rightarrow 242.8$ for remoxipride and $247.0 \rightarrow 84.0,112,218.8$ for raclopride. The skimmer offset was 18 and collision was performed between at fixed voltages between 24 and $45 \mathrm{~V}$. Scan width and scan time were the same as above.

\section{Determination of Fraction Unbound in Plasma}

To determine the free fraction of paliperidone and risperidone in plasma samples, Centrifree Ultrafiltration Devices from Merck Millipore (Amsterdam, The Netherlands) were used to separate the free from the protein bound risperidone and paliperidone in pooled plasma samples. Both the ultrafiltrate and the original pooled plasma sample (without ultrafiltration step) were measured. The free fraction was calculated according to the following Eq. 1:

Free fraction $=\frac{\text { Ultrafiltrate concentration }}{\text { Pooled plasma concentration }}$

For phenytoin and remoxipride, the free fraction in plasma was calculated using a protein binding constant of 91 and $26 \%$ respectively which were obtained from literature $(27,28)$.

\section{Determination of In-Vivo Recovery (retro dialysis) (29)}

The in-vivo recovery of paliperidone, risperidone phenytoin and remoxipride was calculated using the compound concentration in the dialysate $\left(\mathrm{C}_{\text {dial }}\right)$ and in $\mathrm{PF}\left(\mathrm{C}_{\text {in }}\right)$ according to the following Eq. 2:

In vivo recovery $=\frac{C_{\text {in }}-C_{\text {dial }}}{C_{\text {in }}}$

Brain microdialysis data of paliperidone, risperidone, phenytoin and remoxipride were corrected for in-vivo recovery to obtain brain $_{\mathrm{ECF}}$ and CSF data.

The in-vivo recovery and free fraction for the nine compounds are summarized in Table SII.

\section{Human Data}

Table II summarizes the clinical concentration data for acetaminophen and morphine used to assess model performance to predict human concentrations. These data consisted of two clinical studies for acetaminophen and two studies for morphine. All studies were published, except for study 1 for acetaminophen that consists of newly generated data (see in Table II).

\section{Acetaminophen}

Acetaminophen human plasma samples and CSF samples were obtained at Poitier University Hospital. Seven patients who had a traumatic brain injury (TBI) were enrolled in the clinical study. They were treated with a $30 \mathrm{~min}$ intravenous infusion of $1 \mathrm{~g}$ of acetaminophen. CSF samples were collected from a compartment of cerebrospinal fluid in the lateral ventricle $\left(\mathrm{CSF}_{\mathrm{LV}}\right)$ by external-ventricular drainage (EVD) to control the intra-cranial overpressure (named CSF EVD) (30). All clinical studies were conducted according to the Declaration of Helsinki, and written informed consent was obtained from each subject after the approval of the institutional review board at the medical institute. The demographic data is summarized in Table SIII. Acetaminophen concentrations at the start of the study (some patients already received acetaminophen before) were used as an initial value in the plasma compartment. The volume of EVD samples and EVD flow rate during a certain time interval were experimentally determined (Table SIV).

A second human acetaminophen PK dataset (study 2) in plasma and in CSF subarachnoid space $\left(\mathrm{CSF}_{\mathrm{SAS}}\right)$ was obtained from the literature, and was based on patients with nerveroot compression pain (31).

For both datasets, total plasma concentrations for acetaminophen were converted to free plasma concentrations using the free fraction obtained from literature (32).

\section{Morphine}

Morphine human concentration-time profiles in plasma and

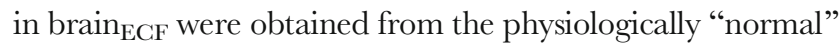
side of the brain and also from the "injured" side of the brain of TBI patients $(33,34)$. For both datasets, the unbound plasma concentrations were already reported in the original publications $(33,34)$.

\section{Software}

The PK analysis was performed using NONMEM version 7.3 (ICON Development Solutions, Hanover, MD, USA) (35). For the brain PK modeling of rat data, the extended least squares estimation method was applied. Other analyses were performed by using the first-order conditional estimation method with interaction (FOCE-I). The compartmental models were defined using the ADVAN6 differential equation solver in NONMEM (35). The plots and the statistical analysis were conducted using $\mathrm{R}$ (Version 3.2.5; R Foundation for Statistical Computing, Vienna, Austria) (36). 
Table II Summary of the Human Acetaminophen and Morphine Data

\begin{tabular}{|c|c|c|c|c|c|}
\hline \multirow[t]{2}{*}{ Study design } & & \multicolumn{2}{|l|}{ Acetaminophen } & \multicolumn{2}{|l|}{ Morphine } \\
\hline & & Study I & Study 2 & Study I & Study 2 \\
\hline Condition of patients & & $\begin{array}{l}\text { human with traumatic } \\
\text { brain injury }\end{array}$ & $\begin{array}{l}\text { human with nerve-root } \\
\text { compression pain }\end{array}$ & $\begin{array}{l}\text { human with traumatic } \\
\text { brain injury }\end{array}$ & $\begin{array}{l}\text { human with traumatic } \\
\text { brain injury }\end{array}$ \\
\hline $\mathrm{Nr}$ of patients & & 7 & I (mean values) & 2 & I \\
\hline Dosage & & I g, 30 min infusion & $\begin{array}{l}2 \mathrm{~g} \text { (propacetamol), } \\
\text { short infusion }\end{array}$ & $10 \mathrm{mg}, 10 \mathrm{~min}$ infusion & $10 \mathrm{mg}, 10 \mathrm{~min}$ infusion \\
\hline \multirow{2}{*}{$\begin{array}{l}\text { Nr of samples } \\
\quad(\text { sampling time, h) }\end{array}$} & plasma & $38(0-6$ h) & II (0-12 h) & $23(0-3 h)$ & II (0-3 h) \\
\hline & brain ECF or CSF & $54(0-5.5$ h) & II (0-13 h) & $74(0-3 h)$ & $37(0-3 h)$ \\
\hline data references & Newly generated & $(31)$ & (34) & (33) & \\
\hline \multicolumn{6}{|l|}{ Data } \\
\hline plasma & & $x$ & $x$ & $x$ & $x$ \\
\hline brain $_{E C F}$ & & & & $\begin{array}{l}\text { X("normal" and "injured" } \\
\text { brain tissue) }\end{array}$ & $\begin{array}{l}\text { X ("normal" and "injured" } \\
\text { brain tissue) }\end{array}$ \\
\hline $\mathrm{CSF}_{\mathrm{EVD}}$ & & $x$ & & & \\
\hline $\mathrm{CSF}_{\mathrm{SAS}}$ & & & $x$ & & \\
\hline$f_{p}^{a}$ & & $85 \%$ & $85 \%$ & - & - \\
\hline$f_{p}$ references & & $(32)$ & $(32)$ & (34) & (33) \\
\hline
\end{tabular}

brain $_{E C F}$ a brain extracellular fluid compartment, CSFEVD a compartment of cerebrospinal fluid in EVD, CSF SAs a compartment of cerebrospinal fluid in subarachnoid space

${ }^{\text {a }}$ free fraction in plasma

\section{Model Development}

Separate models describing plasma and brain concentration-time profiles for all nine compounds were developed whereby plasmaand brain-related parameters were estimated simultaneously. A naïve pooling approach was used (37), i.e. inter-individual variability in each compound's data was not quantified, because of the highly standardized experimental settings combined with the homogeneous nature of the animals within each study.

The structural model that was used as a starting point was based on our previously developed models (23-25). To develop a more generally applicable model structure with parameters that can be precisely estimated across drugs, we systematically assessed the following two model structure characteristics.

First, a combined drug dispersion parameter was estimated to capture the CSF and ECF flow and turbulence flow of the drug molecules $(38,39)$.

Second, drug transfer across the BCSFB was excluded. $\mathrm{SA}_{\mathrm{BCSFB}}$ is 2-15 times smaller than $\mathrm{SA}_{\mathrm{BBB}}(40-42)$, suggesting that drug exchange at BCSFB can be ignored from the model.

We evaluated for each drug the validity of the changes to the basic model with regard to a single or two different flow rates for drug dispersion and drug transport at the BCSFB.

\section{Quantification of Active Drug Transport}

For the 6 compounds, data were obtained using coadministration of inhibitors of active transport. For all these compounds, the effect of the active transport inhibitors was tested on drug exchange at the BBB (Q_L_ECF) and plasma clearance $\left(\mathrm{CL}_{\mathrm{PL}}\right)$, and in combination, as a categorical covariate. (Eq.3)

$P=P_{P A T} \times\left(1+\theta_{\text {cov }} \cdot \operatorname{Cov}\right)$

where $\mathrm{P}_{\mathrm{PAT}}$ represents the parameter including passive and active transport (net transport), $\mathrm{P}$ represents the parameter which takes into account the active transport inhibitors if there is any such effect, Cov is the value of the covariate ( 0 : without an active transport inhibitor, 1: with an active transport inhibitor), $\theta_{\text {cov }}$ represents the effect of the active transport inhibitor.

\section{Model Evaluation}

The systematic inclusion of aforementioned factors was guided by a likelihood ratio test, by an adequate parameter estimation precision, by assessment of the parameter correlation matrix to ensure parameter identifiability, and by the graphical evaluation of plots for observations versus predictions and weighted residuals versus time and versus predictions. The likelihood ratio test is based on the assumption that changes in the NONMEM objective function values (OFV, -2 log likelihood) are asymptotically chisquare distributed. A decrease of $\mathrm{OFV} \geq 3.84$ was considered statistically significant $(p<0.05)$. For a clear 
assessment of model predictions and observations we also computed the following metrics (Eq.4 and 5).

$$
\begin{gathered}
P E=\frac{\Upsilon_{O B S, i j}-\Upsilon_{P R E D, i j}}{\Upsilon_{O B S, i j}-\Upsilon_{P R E D, i j} / 2} \\
S M A P E=\frac{1}{\mathcal{N}} \sum_{k=1}^{\mathcal{N}}|P E| \times 100
\end{gathered}
$$

where PE is a prediction error, and SMAPE is symmetric mean absolute percentage error (43). $\mathrm{Y}_{\mathrm{OBS}, \mathrm{ij}}$ is the $j$ th observation of the $i$ th subject, $\mathrm{Y}_{\mathrm{PRED}, \mathrm{ij}}$ is the $j$ th prediction of the $i$ th subject. $\mathrm{N}$ is number of observations. In the cases where we did not estimate inter-individual variability, e.g. for all brain PK data, $\mathrm{Y}_{\mathrm{PRED}, \mathrm{ij}}$ equals the mean population prediction $\mathrm{Y}_{\mathrm{PRED}_{j} \mathrm{j}}$.

\section{External Model Validation}

Validation of the brain PK model was performed by investigating the quality of the prediction of external rat data. The prediction was done as follows, 1) estimating plasma-related parameters (CLPL, Q PLPER1 $_{\text {PL }}$ and $\mathrm{V}_{\text {PL_PER1 }}$ ) using the external rat plasma data, 2) fixing the brain-related parameters ( $Q_{\text {PL ECF }}, Q_{\mathrm{DIFF}}$, $\mathrm{V}_{\mathrm{ECF}}, \mathrm{V}_{\mathrm{LV}}, \mathrm{V}_{\mathrm{TFV}}, \mathrm{V}_{\mathrm{CM}}$, and $\mathrm{V}_{\mathrm{SAS}}$ ) to the values which were estimated from the brain PK model and 3) predicting the brain $_{\mathrm{ECF}}$ or CSF concentrations using estimated rat plasmarelated parameters and fixed brain-related parameters.

\section{Plasma PK Analysis of External Rat Data}

The plasma-related parameters including inter-individual variability on these parameters and residual errors were estimated using the external rat plasma data. We used a mixed effects modeling approach to investigate the predictability of the brain concentration based on each plasma concentration. The same plasma model structure, which was obtained from the brain PK model was applied for each compound. Interindividual variability were tested on each PK parameter using an exponential model (Eq. 6).

$\theta_{i}=\theta \times e^{\eta_{\mathrm{i}}}$

where $\theta_{\mathrm{i}}$ represents the parameters of the $i$ th subject, $\theta$ represents the population mean value of the parameter, and $\eta_{i}$ is the random effect of the $i$ th subject under the assumption of a normal distribution with a mean value of 0 and variance of $\omega^{2}$.

A proportional error model and the mixed error model (Eq. 7-8) were tested for the residual errors:

$$
\begin{aligned}
C_{i j} & =\Upsilon_{P R E D, i j} \times\left(\mid+\varepsilon_{i j}\right) \\
C_{i j} & =\Upsilon_{P R E D, i j} \times\left(\mid+\varepsilon_{\mid, i j}\right)+\varepsilon_{2, i j}
\end{aligned}
$$

where $\mathrm{C}_{\mathrm{ij}}$ represents the $j$ th observed concentration of the $i$ th subject, $Y_{\text {PRED,ij }}$ represents the $j$ th predicted concentration of the $i$ th subject, and $\varepsilon_{\mathrm{ij}}$ is the random effect of the $j$ th observed concentration of the $i$ th subject under the assumption of a normal distribution with a mean value of 0 and variance of $\sigma^{2}$.

Model selection was guided by a likelihood ratio test with $p<0.05$ and by the precision of the parameter estimates.

\section{Handling of the Brain-Related Parameter Values}

For $Q_{\text {PL_ECF, }} Q_{\text {DIFF, the same values, which were estimated }}$ from the brain PK model, were used for acetaminophen and remoxipride, respectively. $\mathrm{V}_{\mathrm{ECF}}, \mathrm{V}_{\mathrm{LV}}, \mathrm{V}_{\mathrm{TFV}}, \mathrm{V}_{\mathrm{CM}}$, and $\mathrm{V}_{\mathrm{SAS}}$ are system-specific parameters, therefore, the same rat physiological values were used, indicated in Table III.

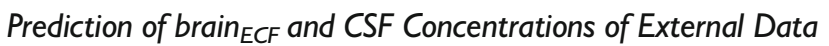

Simulations were performed 200 times for each compound. The 95\% prediction interval (using the calculated $2.5 \%$ tile and $97.5 \%$ tile) and the median of the simulated concentrations were plotted together with the external data. Accuracy of the mean population prediction for brain PK data was evaluated with SMAPE mentioned above (Eq. 5).

\section{Translation of the Model to Humans}

The translational prediction was performed by the following steps, 1) estimating plasma-related parameters $\left(\mathrm{CL}_{\mathrm{PL}}, \mathrm{Q}_{\mathrm{PL}}\right.$ PER1 $V_{\text {PL }}$ and $V_{\text {PL_PER1 }}$ ) using human plasma data, 2) replacing brain-related system-specific parameters $\left(\mathrm{V}_{\mathrm{ECF}}, \mathrm{V}_{\mathrm{LV}}\right.$, $\mathrm{V}_{\mathrm{TFV}}, \mathrm{V}_{\mathrm{CM}}$ and $\mathrm{V}_{\mathrm{SAS}}$ ) by human values, 3) applying allometric scaling to the brain-related drug-specific parameters which were estimated with the rat in-vivo data Q $_{\text {PL_EGF }}$ and Q DIFF), 4) adding clinical sampling procedure related fixed parameters which were obtained from the EVD into the model ( $Q_{\text {LV EVD }}$ and $\left.\mathrm{V}_{\mathrm{EVD}}\right)$ and 5) predicting the brain ECF $_{\text {and }}$ CSF concentrations using estimated human plasma PK parameters, replacing system-specific parameters, scaling drugspecific parameters and using clinical sampling procedure related fixed parameters. The details of the translational methods for each parameter are explained in Fig. 2.

\section{Human Plasma PK Analysis}

Plasma-related parameters including inter-individual variability and residual errors were estimated using the human data using the Eqs. 6-8. A 1-compartment, 2-compartment and 3compartment model were tested. Model selection was guided by a likelihood ratio test with $p<0.05$, by the precision and correlation between parameter estimates and by the graphical evaluation of plots for observations versus predictions and weighted residuals versus time and versus predictions. 


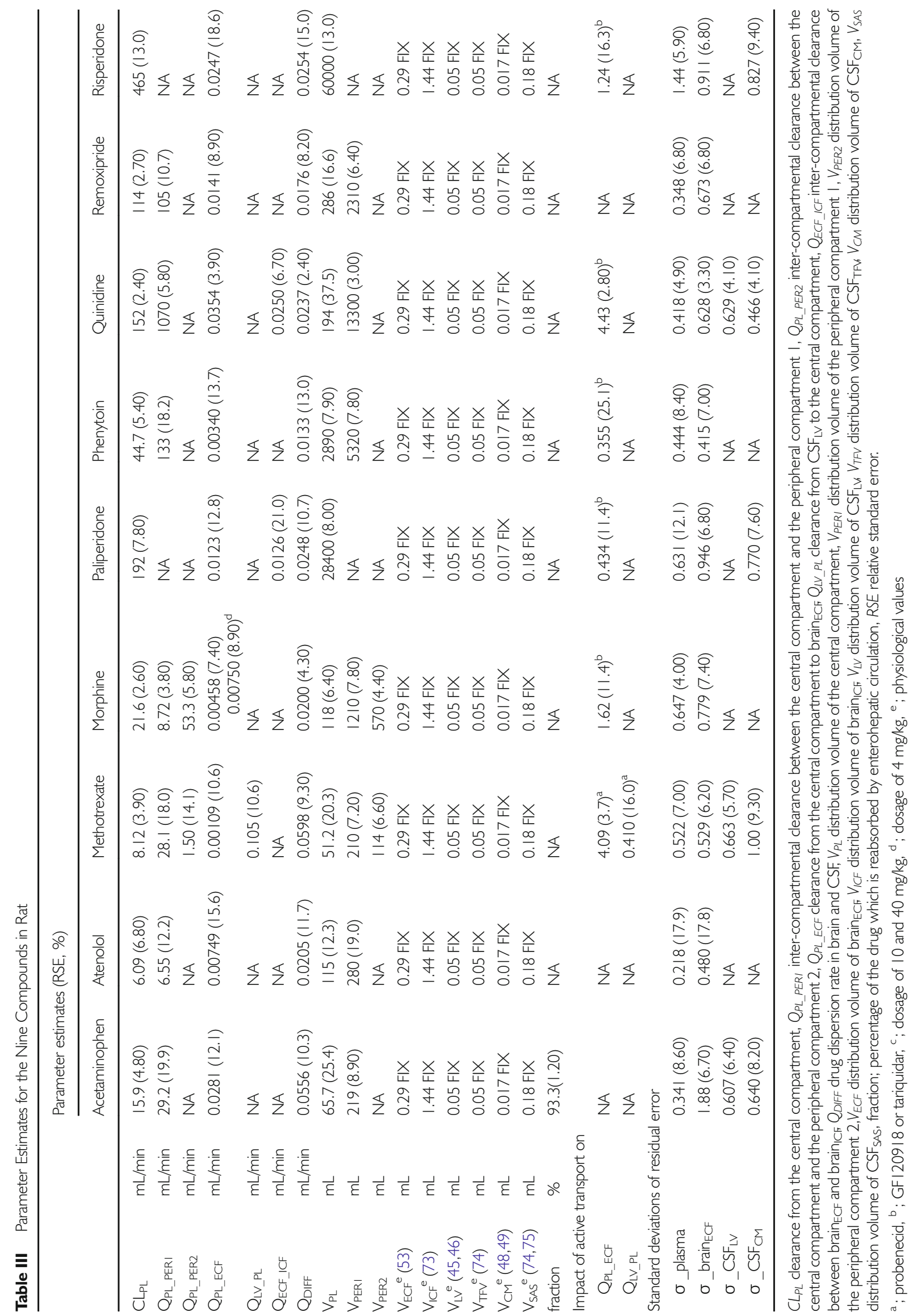


Fig. 2 The brain PK model structure and translational methods for each parameter. The brain PK model consists of plasma, brain ${ }_{E C F}$ brain ${ }_{\text {ICF }}$ CSF $_{\text {LV }}$ CSF TFV $_{\text {CSF }}$ CM and $\mathrm{CSF}_{\mathrm{SAS}}$, which consists of 4 different categories parameters (colors). The scaling method on each parameter is indicated with color coding.

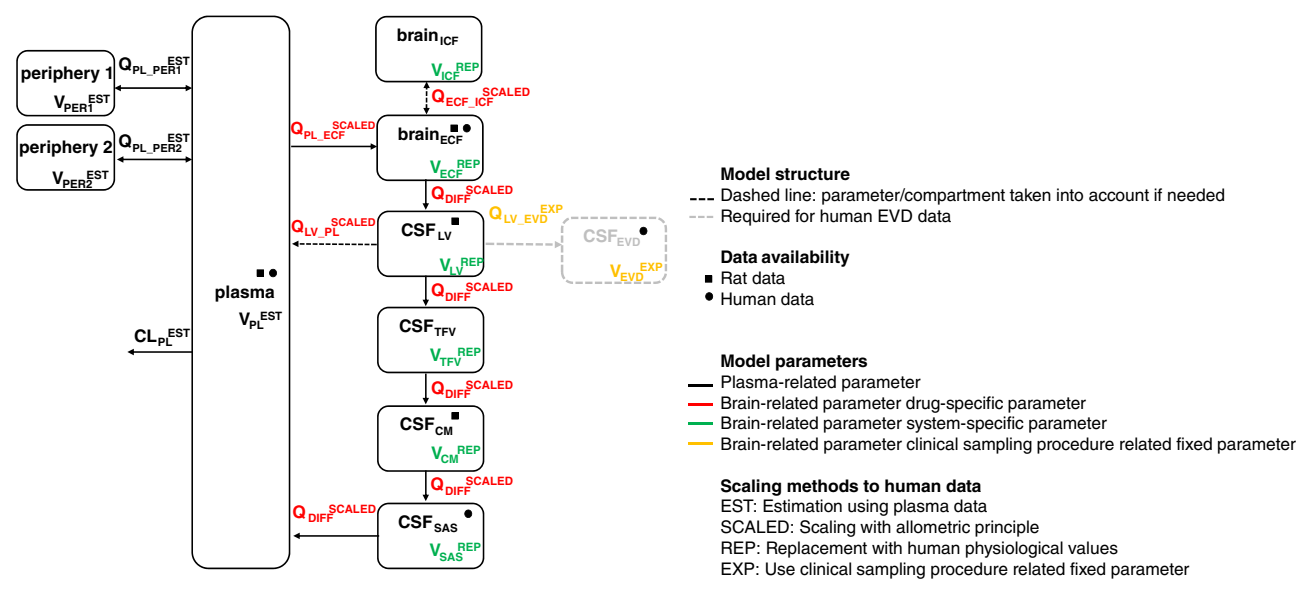

\section{Replacement of the System-Specific Parameters}

System-specific parameters in the brain distribution rat model $\left(\mathrm{V}_{\mathrm{ECF}}, \mathrm{V}_{\mathrm{LV}}, \mathrm{V}_{\mathrm{TFV}}, \mathrm{V}_{\mathrm{CM}}\right.$ and $\mathrm{V}_{\mathrm{SAS}}$ ) were replaced with the human physiological values, which are available from literature (44-50) (see Table IV).

\section{Scaling of the Drug-Specific Parameters}

Drug-specific parameters (CLPL_ECF and Q Q to human values using allometric principles following Eq. 9 (18).

$P_{\text {human }}=P_{\text {rat }} \times\left(\frac{B W_{\text {human }}}{B W_{\text {rat }}}\right)^{0.75}$

where $\mathrm{P}_{\text {human }}$ is the scaled human parameter, $\mathrm{P}_{\text {rat }}$ is the estimated rat parameter from the model, $\mathrm{BW}_{\text {human }}$ is the average human body weight $(70 \mathrm{~kg})$, and $\mathrm{BW}_{\text {rat }}$ is the average rat body weight (250 g).

\section{Adding Clinical Sampling Procedure Related Fixed Parameters}

In addition to those parameters which were used in the rat brain PK model, we have data obtained from the EVD approach, therefore the EVD compartment was added into the translated brain distribution model (see Fig. 2). To describe the PK of acetaminophen in the EVD compartment, the values of flow rate from $\mathrm{CSF}_{\mathrm{LV}}$ to $\mathrm{CSF}_{\mathrm{EVD}}\left(\mathrm{Q}_{\mathrm{LV} \text { EVDD }}\right)$ and the volume of EVD compartment $\left(\mathrm{V}_{\mathrm{EVD}}\right)$ were added into the

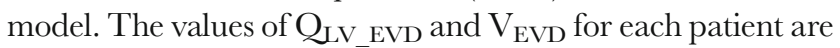
obtained from EVD approach and available in Table SIV.

\section{Prediction of Human brain ECF $_{\text {and CSF Concentrations }}$}

Simulations were performed using the same methods as we mentioned for the external model validation.

\section{RESULTS}

The analysis work flow is depicted in Fig. 3. The developed multi-compartmental brain PK model adequately described the data for the nine compounds, as can be observed from the selected observed and predicted concentration-time profiles (Fig. 4a) and the prediction error plots for all of the nine compounds (Fig. 4b). The prediction errors were mostly within two standard deviations of zero, i.e. no systematic differences between observations and predictions were found. No specific trend across time, also with respect to the presence or absence of active transport inhibitors, were observed. More extensive plots for individual observations versus predictions and weighted residuals versus time across drugs, dose levels and active transport inhibitors, are provided in the supplemental material (Figure S1 and S2).

We identified a generally applicable model structure (Fig. 2) with physiologically relevant compartments. The final model consists of plasma, brain ${ }_{\mathrm{ECF}}$, brain intracellular fluid

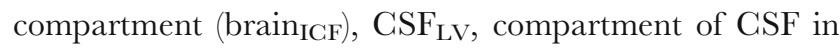
third and fourth ventricle $\left(\mathrm{CSF}_{\mathrm{TFV}}\right)$, compartment of $\mathrm{CSF}$ in cisterna magna $\left(\mathrm{CSF}_{\mathrm{CM}}\right)$ and $\mathrm{CSF}_{\mathrm{SAS}}$, which included processes for drug exchange at the BBB (QPL_ECF) and drug dispersion through brain ECF $_{\text {and CSF compartments (Q }}$ DIFF). The parameter estimates were obtained with good precision, and are summarized in Table III.

A single drug dispersion rate ( $\left.Q_{\mathrm{DIFF}}\right)$ was shown to be sufficient for describing the sum of the drug distribution in the brain $_{\mathrm{ECF}}$ and CSF for the nine compounds. QDIFF was comparable among the compounds, and ranged between $0.0598 \mathrm{~mL} / \mathrm{min}$ for methotrexate to $0.0133 \mathrm{~mL} / \mathrm{min}$ for phenytoin, and could be precisely identified (RSE $<15.0 \%$, suggesting this parameter could be potentially considered to represent a system-specific parameter.

The parameter representing drug transfer at the BBB (QPL_EGF) was critical to quantify drug exchange between blood and brain. QPL ECF was substantially different between 
Table IV Parameter Values used for the Translational Prediction to Humans

\begin{tabular}{|c|c|c|c|c|}
\hline & \multirow[t]{2}{*}{ Translational methods } & \multirow[t]{2}{*}{ Unit } & \multicolumn{2}{|c|}{ Parameter estimates (RSE, \%) } \\
\hline & & & Acetaminophen & Morphine \\
\hline \multicolumn{5}{|c|}{ Plasma-related parameters } \\
\hline$C_{P L}$ & estimation from human PK data & $\mathrm{mL} / \mathrm{min}$ & $562(20.1)$ & $3070(15.8)$ \\
\hline QPL_PERI & estimation from human PK data & $\mathrm{mL} / \mathrm{min}$ & $2060(31.1)$ & $3030(0.60)$ \\
\hline$V_{P L}$ & estimation from human PK data & $\mathrm{mL}$ & $9880(41.1)$ & $16000(35.3)$ \\
\hline$V_{\text {PERI }}$ & estimation from human PK data & $\mathrm{mL}$ & $51900(18.3)$ & $95400(2.50)$ \\
\hline \multicolumn{5}{|c|}{ Brain-related parameters } \\
\hline \multicolumn{5}{|c|}{ Drug-specific parameters } \\
\hline QPL_ECF & allometric scaling & $\mathrm{mL} / \mathrm{min}$ & 1.92 FIX & $0.513 \mathrm{FIX}$ \\
\hline$Q_{\text {DIFF }}$ & allometric scaling & $\mathrm{mL} / \mathrm{min}$ & $3.81 \mathrm{FIX}$ & I.37 FIX \\
\hline \multicolumn{5}{|c|}{ System-specific parameters } \\
\hline$V_{E C F}^{a}(44)$ & replacement & $\mathrm{mL}$ & $240 \mathrm{FIX}$ & $240 \mathrm{FIX}$ \\
\hline$V_{L V}{ }^{a}(45-47)$ & replacement & $\mathrm{mL}$ & $22.5 \mathrm{FIX}$ & $22.5 \mathrm{FIX}$ \\
\hline $\mathrm{V}_{\text {TFV }}{ }^{\mathrm{a}}(45-47)$ & replacement & $\mathrm{mL}$ & $22.5 \mathrm{FIX}$ & $22.5 \mathrm{FIX}$ \\
\hline$V_{C M}{ }^{a}(48,49)$ & replacement & $\mathrm{mL}$ & $7.5 \mathrm{FIX}$ & $7.5 \mathrm{FIX}$ \\
\hline$V_{S A S}^{a}(50)$ & replacement & $\mathrm{mL}$ & $90 \mathrm{FIX}$ & $90 \mathrm{FIX}$ \\
\hline \multicolumn{5}{|c|}{ Clinical sampling procedure related fixed parameters } \\
\hline $\begin{array}{l}\text { QLV_EVD } \\
V_{E V D}\end{array}$ & $\begin{array}{l}\text { use the fixed parameter } \\
\text { use the fixed parameter }\end{array}$ & $\begin{array}{l}\mathrm{mL} / \mathrm{min} \\
\mathrm{mL}\end{array}$ & \multicolumn{2}{|c|}{ values are in supplemental Table IV } \\
\hline \multicolumn{5}{|c|}{ Standard deviations of inter-individual variability (estimated from human PK data) } \\
\hline$\omega_{-} C_{P L}$ & & & $0.490(30.2)$ & $0.27 \mid(19.9)$ \\
\hline$\omega_{-} Q_{P L}$ PERI & & & NA & NA \\
\hline$\omega_{-} V_{P L}$ & & & NA & $0.596(20.0)$ \\
\hline$\omega_{-} V_{\text {PERI }}$ & & & $0.235(22.5)$ & NA \\
\hline \multicolumn{5}{|c|}{ Standard deviations of residual error (estimated from human PK data) } \\
\hline o_plasma & & & $0.250(8.20)$ & $0.0960(22.9)$ \\
\hline
\end{tabular}

$C_{P L}$ clearance from the central compartment, $Q_{P L}$ PERI inter-compartmental clearance between the central compartment and the peripheral compartment I, $V_{P L}$ distribution volume of the central compartment, $V_{P E R I}$ distribution volume of the peripheral compartment I, $Q_{P L}$ ECF clearance from the central compartment to brain ${ }_{E C F} Q_{D I F F}$ drug diffusion rate in brain and CSF, $V_{E C F}$ distribution volume of brain ${ }_{E C F} V_{L V}$ distribution volume of $C S F_{L V} V_{T F V}$ distribution volume of CSF $V_{C M}$ distribution volume of $C_{C S}$, $V_{S A S}$ distribution volume of $C_{S F} F_{S A S}, Q_{L V} E V D$ flow from $C S F_{L V}$ to $C S F_{E V D}, V_{E V D}$ volume of CSF ${ }_{\mathrm{EVD}}$

a ; physiological values drugs, ranging from $0.0354 \mathrm{~mL} / \mathrm{min}$ for quinidine to $0.00109 \mathrm{~mL} / \mathrm{min}$ for methotrexate.

On the other hand, drug exchange at BCSFB was identified only for methotrexate, and could not be identified for the other 8 compounds. For methotrexate, the efflux transport at BCSFB ( $\mathrm{Q}_{\mathrm{LV} \text { PL }}$ ) was $0.105 \mathrm{~mL} / \mathrm{min}$.

Among the nine compounds, clearance between brain ${ }_{\mathrm{ECF}}$ and brain ${ }_{\mathrm{ICF}}\left(\mathrm{Q}_{\mathrm{ECF} \text { ICF }}\right)$ could be estimated for paliperidone and quinidine: $\mathrm{Q}_{\mathrm{ECF}}$ ICF is $0.0250 \mathrm{~mL} / \mathrm{min}$ for quinidine, and $0.0126 \mathrm{~mL} / \mathrm{min}$ for paliperidone, implying for quinidine a slightly faster uptake into brain ${ }_{\text {ICF }}$ after crossing the BBB (Table III).

For morphine, brain $_{\mathrm{ECF}}$ concentration displayed a nonlinear relationship with dose and plasma concentrations. A categorical dose effect was therefore estimated. Continuous linear or nonlinear concentration-dependent effects to account for this effect were not supported by the data.
No statistically significant impact of P-gp and the combination of MRPs, OATs and OATPs on CLPL could be identified, whereas those transporters were identified to act as efflux transporters at the BBB for our compounds. The P-gp function was quantified on the data of morphine, paliperidone, phenytoin, quinidine, and risperidone, and the impact of the combination of MRPs, OATs and OATPs was quantified on the data of methotrexate, as a categorical covariate on $Q_{\text {PL ECF. The pres- }}$ ence of P-gp inhibitors increased the QPL_ECF values of morphine, paliperidone, phenytoin, quinidine, and risperidone by $162,43.4,35.5,443$ and $124 \%$ respectively. The presence of the inhibitor of MRPs, OATs and OATPs increased the QPL ECF values of methotrexate by $409 \%$.

The developed model adequately predicted the external rat acetaminophen and remoxipride data. Figure 5 presents the prediction results for the external rat data of 
Data

(black: published data, blue: new data)

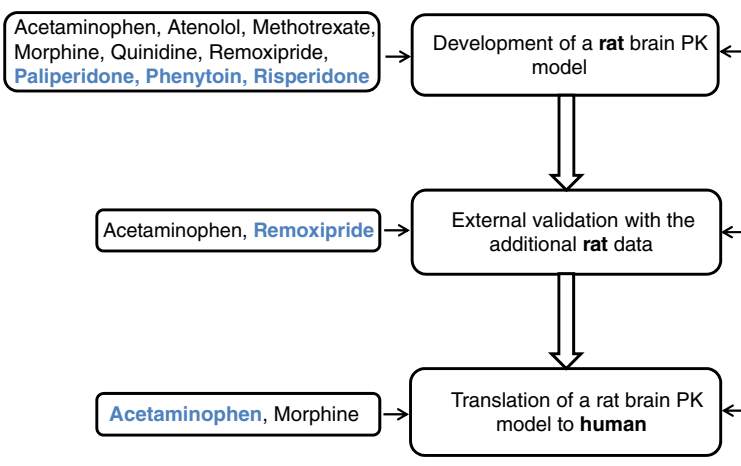

Methodology

Plasma-related parameters: EST

Brain-related system-specific parameters: FIX

Brain-related drug-specific parameters: EST

Plasma-related parameters: EST

Brain-related system-specific parameters: FIX

Brain-related drug-specific parameters: FIX

Plasma-related parameters: EST

Brain-related system-specific parameters: REP

Brain-related drug-specific parameters: SCALED

Brain-related parameter clinical sampling procedure related fixed parameter: EXP

EST: estimation using plasma data

FIX: fixed to the values obtained from the model development

SCALED: scaling with allometric principle

REP: replacement with human physiological value

EXP: use clinical sampling procedure related fixed parameter

Fig. 3 Schematic flow chart of the analysis.

acetaminophen and remoxipride using the developed multicompartmental brain PK model. Prediction of the acetaminophen concentration-time profile in brain $_{\mathrm{ECF}}$ using the final model captured the external acetaminophen concentration in brain $_{\mathrm{ECF}}$ well (SMAPE $<61 \%$ ). Prediction of the remoxipride concentration-time profile in brain $_{\mathrm{ECF}}, \mathrm{CSF}_{\mathrm{LV}}$ and $\mathrm{CSF}_{\mathrm{CM}}$ using the final model also captured the external remoxipride concentrations in brain $_{\mathrm{ECF}}, \mathrm{CSF}_{\mathrm{LV}}$ and $\mathrm{CSF}_{\mathrm{CM}}$ concentrations well (SMAPE $<67,77,56 \%$, respectively).

The model was successfully scaled to predict concentrationtime profiles of acetaminophen and morphine in human brain compartments. Table IV summarizes the parameter values that were used for the prediction of human plasma,

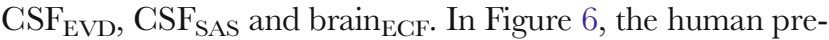
dictions versus human observations are depicted. The acetaminophen human $\mathrm{CSF}_{\mathrm{SAS}}$ concentration in the patients with nerve-root compression pain and $\mathrm{CSF}_{\mathrm{EVD}}$ concentration in the patients with TBI were predicted relatively well (SMAPE $<90$ and 66\% respectively), even though there is a slightly faster elimination in $\mathrm{CSF}_{\mathrm{SAS}}$. Morphine brain ECF $_{\text {con- }}$ centrations in the physiologically "normal" brain tissue of TBI patients were predicted very well (SMAPE $<35 \%$ ). However, morphine brain ECF $_{\text {concentrations were underpredicted }}$ when the brain ${ }_{\mathrm{ECF}}$ concentrations were taken from "injured" brain tissue of TBI patients (SMAPE $<56 \%$ ).

\section{DISCUSSION}

The developed multi-compartmental brain PK model could describe the data of the nine compounds in the rat adequately in the absence and presence of active transport blockers (Fig. 4). After scaling of the model, human brain concentration-time profiles of acetaminophen and morphine could be adequately predicted in several physiological compartments under normal physiological conditions.

The model structure we have derived differs from the ones published earlier by: (i) a combined drug dispersion parameter was estimated to capture the CSF and brain ${ }_{\mathrm{ECF}}$ flow and turbulence flow of the drug molecules; and (ii) drug transfer across the BCSFB was excluded (23-25). The final model has four different CSF compartments. This model is developed to predict human brain concentration profiles using rat data. In our analysis, rat data was sampled from $\mathrm{CSF}_{\mathrm{LV}}$ and $\mathrm{CSF}_{\mathrm{CM}}$. Since in rats it is anatomically easier to access the $\mathrm{CSF}_{\mathrm{CM}}$ compartment to obtain drug concentration by microdialysis and by the cisternal puncture methods, there are more data available from $\mathrm{CSF}_{\mathrm{CM}}$ (51). Through keeping the $\mathrm{CSF}_{\mathrm{CM}}$ compartment in the model structure, it will be easier to apply the model to additional compounds' data obtained in animals. Furthermore, substantial differences between CNS compartments may exist, such as a concentration difference between $\mathrm{CSF}_{\mathrm{LV}}$ and $\mathrm{CSF}_{\mathrm{CM}}$ for methotrexate and quinidine in rat $(24,25)$. Thus, to predict the drug target site concentration, the location of the CSF sampling site should be taken into account. For human, in clinical studies most CSF samples are taken from other CSF compartments, such as $\mathrm{CSF}_{\mathrm{SAS}}$ and $\mathrm{CSF}_{\mathrm{LV}}$ where samples are taken by EVD. Hence, we think that our model structure is a minimal, necessary model structure for translation.

We found that the brain intracellular fluid compartment

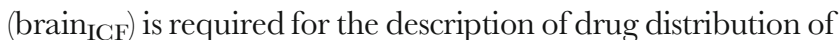
quinidine and paliperidone, and likely associated with the lipophilic basic nature of quinidine ( $\mathrm{pKa} 13.9, \log \mathrm{P} 3.4$ ) and paliperidone (pKa 13.7, $\log$ P 1.8). For other compounds with a less distinct lipophilic-basic nature, such as for 
a

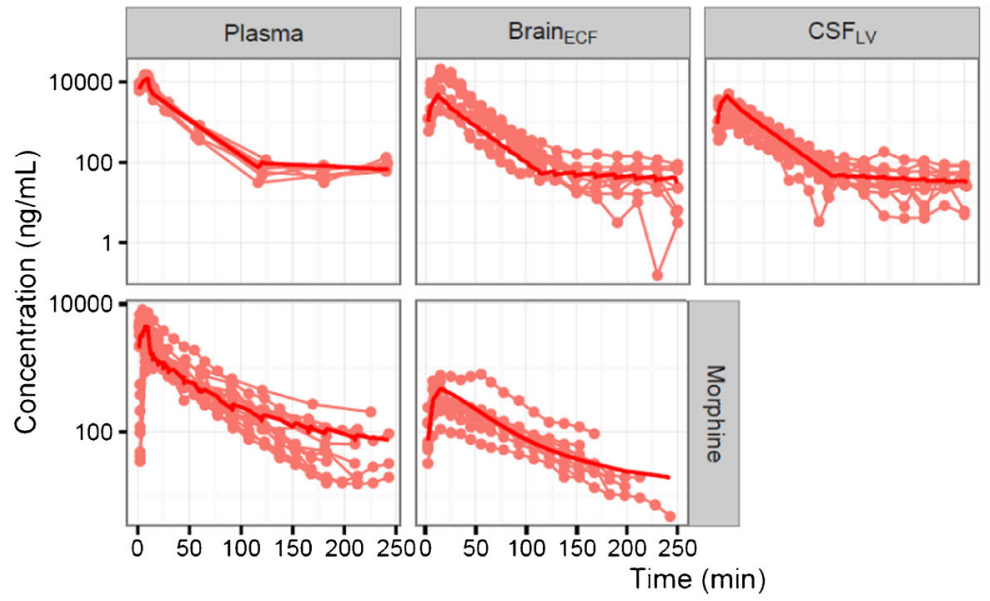

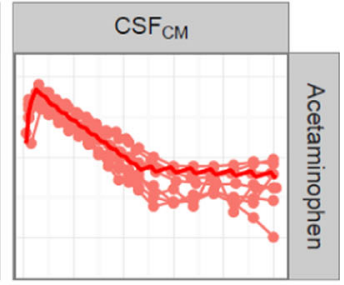

b

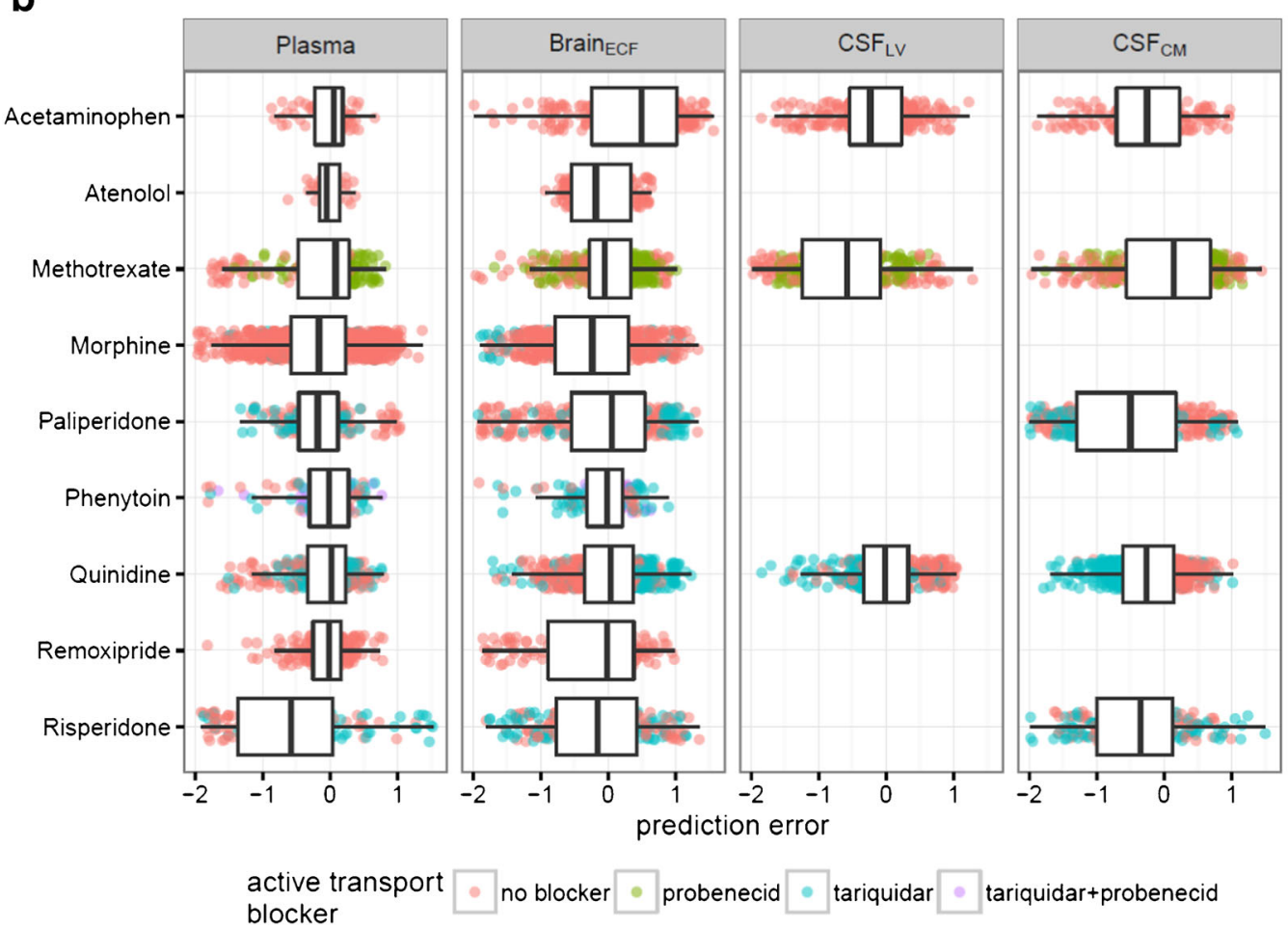

Fig. 4 Prediction of the multi-compartmental brain PK model. (a) Individual observed drug concentrations (lines and circles) and mean model prediction (solid lines). Unbound concentration ( $\mathrm{ng} / \mathrm{mL}$ ) versus time ( $\mathrm{min}$ ) profiles for acetaminophen and morphine. (b) Box-whisker plots for the prediction errors across all nine drugs evaluated. The plots were stratified by brain compartments (panels) and by active transport blockers (colors).

acetaminophen and phenytoin, we have shown that brain ICF $_{\text {F }}$ was not required for the description of concentration-time profiles in the brain. However, for a generally applicable brain PK model, inclusion of this compartment would still be required since prediction of intracellular drug concentrations would be of relevance for GNS drug development as well as prediction of extracellular drug concentrations. Our model and the microdialysis methodology used only allow quantification of extracellular concentrations. However, in combination with PBPK modeling based principles to predict intracellular partitioning, our model will be of significant relevance as it provides the required predictions for unbound extracellular drug concentration kinetics.

A drug exchange parameter across the BCSFB ( $\left.\mathrm{Q}_{\mathrm{LV}} \mathrm{PL}\right)$ was identified for methotrexate only, even though it could not be identified for the other 8 compounds. This suggests that an additional efflux transporter might be present at the BCSFB for which methotrexate is a substrate. It is known that methotrexate is indeed a substrate of various transporters, such as RFC1, MRP, BCRP, OATP and OAT transporters (25), which are not involved in the drug transfer of the other 8 compounds. This result indicates that drug transport at 


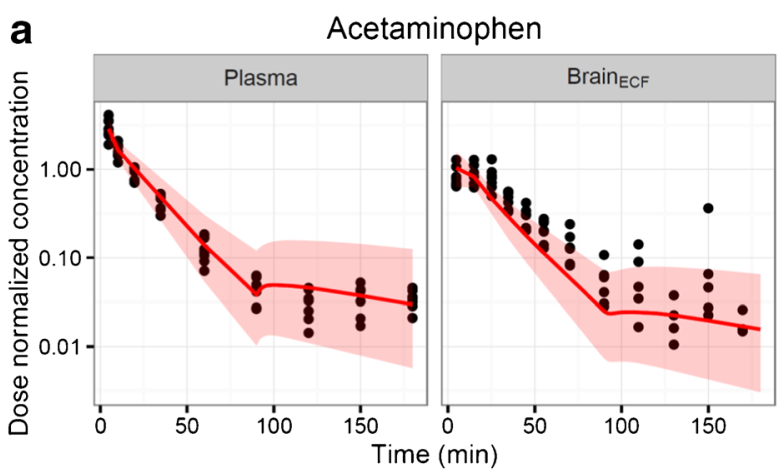

b

Remoxipride
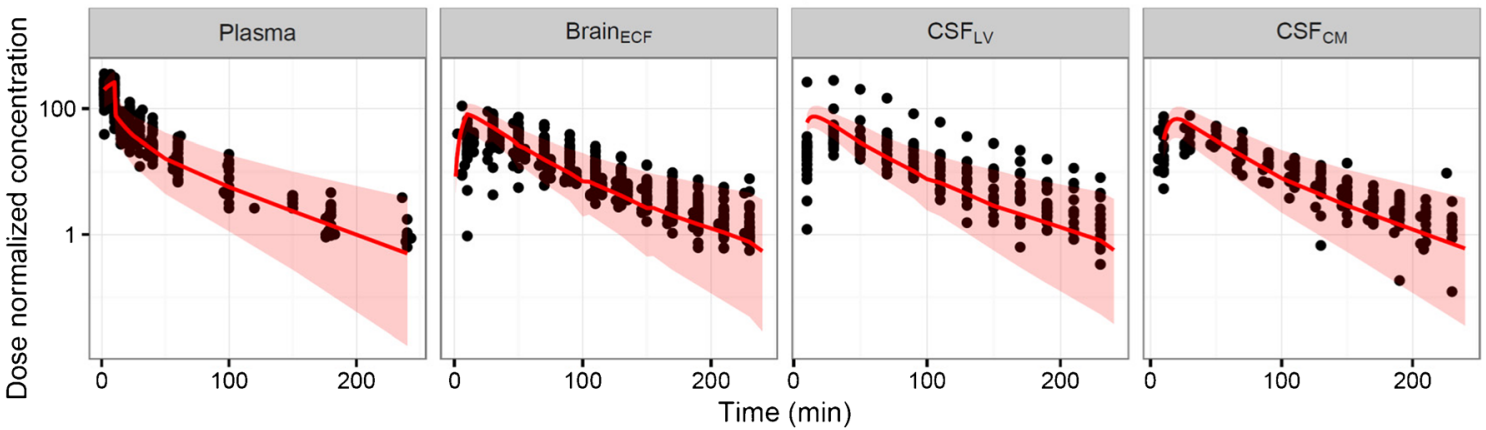

Fig. 5 Model prediction versus external acetaminophen and morphine data in rat. Individual concentration-time profile of the external data (circles) and prediction from the brain PK model (red lines: median, shaded area is 95\% prediction interval). (a) Acetaminophen data were obtained after 200 mg administration, (b) remoxipride data were obtained from the dose group of $0.7,5.2$ and $14 \mathrm{mg} / \mathrm{kg}$. The $x$-axis represents the time in minutes and the $y$-axis represents the dose-normalized acetaminophen and remoxipride concentration. The panels are stratified by brain compartments and compounds.

Fig. 6 Human brain HCF $_{\text {and }}$ CSF concentration-time profiles (circles) and prediction from the translational model (red lines: median, shaded area is $95 \%$ prediction interval). (a) Acetaminophen data was obtained from plasma, $\mathrm{CSF}_{\mathrm{SAS}}$ and $\mathrm{CSF}_{\mathrm{EVD}}$, (b) morphine data was obtained from plasma and brain ECF $_{\text {in }}$ "normal" brain and "injured" brain. The $x$-axis represents the time in minutes and the $y$-axis represents the acetaminophen and morphine concentration in $\mathrm{ng} / \mathrm{ml}$. The panels are stratified by brain compartments and brain conditions.

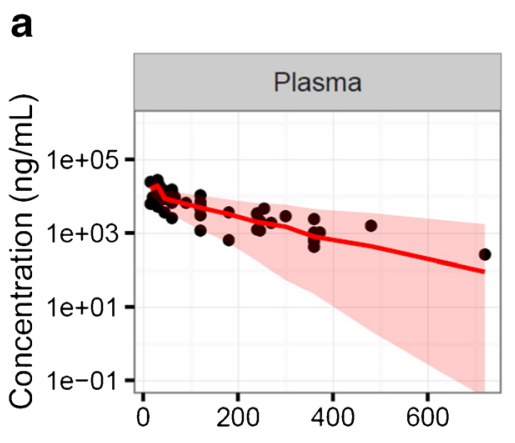

\section{Acetaminophen}

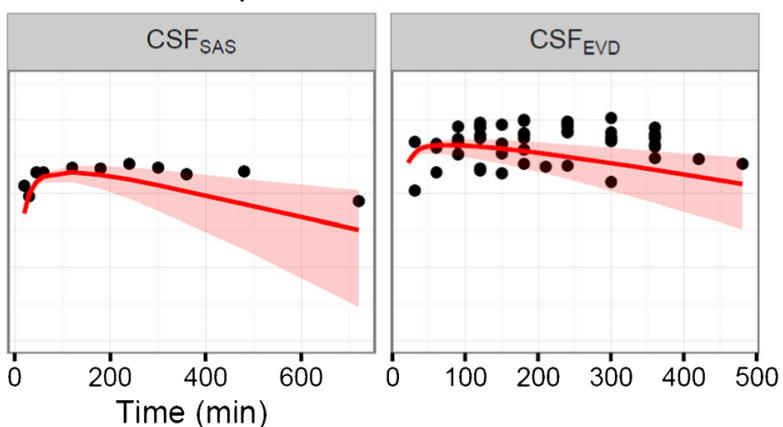

b

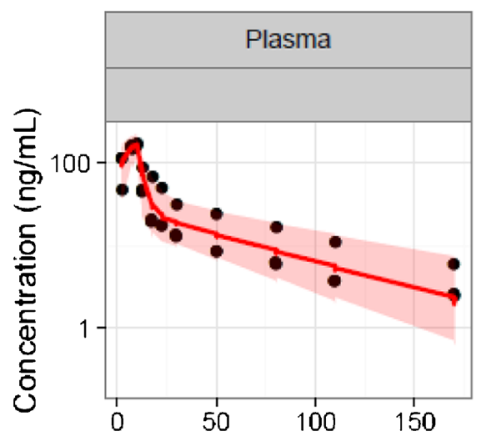

Morphine

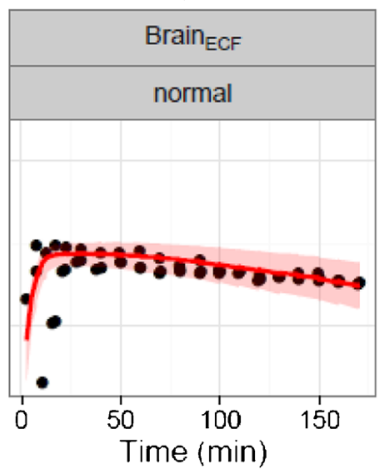


BCSFB still needs to be investigated using data on compounds which are substrates for those transporters. The current model delineates the process that can be used to arrive to the bestperforming model for such drugs. We took care to design the modeling process such that the total number of models that need to be fitted is minimal.

We identified a drug dispersion rate parameter that captures

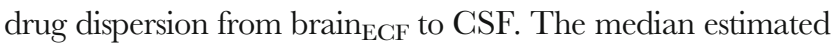
drug dispersion flow was $0.0237 \mathrm{~mL} / \mathrm{min}$. The magnitude of the drug dispersion rate was approximately ten times faster than the reported physiological CSF flow rate alone (52), and about 100 times faster than the reported physiological brain ECF bulk flow rate $(53,54)$. Since similar values across drugs were identified, the parameter may be considered a systemspecific parameter that could be fixed in further analyses (see Table III), to allow for estimation of other processes of interest.

P-gp transport for quinidine, risperidone, paliperidone, morphine and phenytoin was confirmed as efflux transporter at the BBB which were in line with literature $(55,56)$. P-gp transporter effects were not identified at the BCSFB for these 5 P-gp substrates, i.e. CSF concentrations for these compounds were well-described solely by the BBB mediated Pgp transport. The role and contribution of $\mathrm{P}$-gp transporters at the BCSFB is still inconsistent, and both efflux and influx processes have been reported (57-59). Our results however suggest that the function of P-gp may be ignored, since its potential magnitude likely is negligible compared to transport at the BBB, and drug dispersion processes prevail. Nonetheless, overall, we envision that the combination of our dynamical modeling approach with the incorporation of in-vitro assays to characterize active transport across the BBB or BCSFB, may be a fruitful direction to further characterize and disentangle the precise contribution to the brain drug disposition of different drug transport.

The developed model adequately predicted the external acetaminophen and remoxipride rat data, confirming the reliability of the model. Both of these drugs were also used for model development, but the experiments were different and applied somewhat different designs. Since we aimed to generate mean predictions, the variation in numbers of animals is expected to result in limited bias in the modeling. Furthermore, sampling time points were very informatively distributed and any inter-experimental differences in these time points are therefore also considered to be of limited impact on model development. The external validation results indicated that the model is robust with respect to variations in experimental designs and conditions (i.e. the number of rats, sampling times, infusion times, and flow rates of microdialysis).

We consider the developed model structure suited for translational predictions of human brain (target site) concentrations such as required during drug development. The predictive performance in human data ranged between SMAPE of 35-90\%. Even though errors $<90 \%$ may appear large, such $<$ two-fold error is not considered unacceptable when compared to for instance QSAR studies, which are used to predict unbound brain partition coefficients of drugs in drug development (60,61). Secondly, the prediction error is likely inflated because of the use of human data obtained from patients with traumatic brain injury or with nerve-root compression pain. Therefore, larger variability in their physiological condition is expected.

Body weight in combination with allometric scaling was used to scale the parameters to humans, and this resulted in adequate predictions of human brain concentrations for physiologically "normal" brains. Different translational methods for estimation of CNS PK parameters have been reported in the literature. For instance, system-based scaling was applied using volume of brain tissue or brain endothelial surface area $(25,62)$, but allometric scaling using body weight (our approach) was supported by work from others in the literature (63-66). Based on our current approach, reasonable predictions were obtained. Therefore, we suggest that the allometric scaling approach may indeed be appropriate although it would be worthwhile to investigate alternative approaches.

Our model was developed based on healthy rats and then translated to human data that was partly based on patients with severe brain injuries. Indeed, observed human morphine concentrations in brain ECF $_{\text {obtained from }}$ the "injured" side of the brain of the TBI patients was higher than the prediction from the translational model (Fig. 6). It is known that the BBB permeability is increased after TBI, which may be the reason for the underprediction of our translational model for those data $(67,68)$. Therefore, for predictions in patients with pathological conditions that alter the integrity of $\mathrm{BBB}$ or BCSFB barriers, or brain fluid flows, our model should be further extended with additional physiological details.

\section{CONCLUSION}

A multi-compartmental brain PK model structure was developed across a wide range of drugs with different physicochemical properties. The model structure was shown to be of relevance for the scaling of brain concentrations in humans. As such, the developed model structure can be used to inform the prediction of relevant target site concentrations in humans and aid in the translational development of CNS targeted drugs.

\section{ACKNOWLEDGMENTS AND DISCLOSURES}

This research article was prepared within the framework of project no. D2-501 of the former Dutch Top Institute 
Pharma, currently Lygature (Leiden, the Netherlands; www. lygature.org). The authors have no conflicts of interest that are directly relevant to the contents of this research article.

Open Access This article is distributed under the terms of the Creative Commons Attribution 4.0 International License (http://creativecommons.org/licenses/by/4.0/), which permits unrestricted use, distribution, and reproduction in any medium, provided you give appropriate credit to the original author(s) and the source, provide a link to the Creative Commons license, and indicate if changes were made.

\section{REFERENCES}

1. Kola I, Landis J. Can the pharmaceutical industry reduce attrition rates. Nat Rev Drug Discov. 2004;3:711-5.

2. Hurko O, Ryan JL. Translational research in central nervous system drug discovery. J Am Soc Exp Neurother. 2005;2(4):67 1-82.

3. Cook D, Brown D, Alexander R, March R, Morgan P, Satterthwaite $\mathrm{G}$, et al. Lessons learned from the fate of AstraZeneca's drug pipeline: a five-dimensional framework. Nat Rev Drug Discov Nat Publ Group. 2014;13(6):419-31.

4. Danhof M, de Jongh J, De Lange ECM, Della Pasqua O, Ploeger BA, Voskuyl RA. Mechanism-based pharmacokinetic-pharmacodynamic modeling: biophase distribution, receptor theory, and dynamical systems analysis. Annu Rev Pharmacol Toxicol. 2007;47(1):357-400.

5. De Lange EGM. The mastermind approach to GNS drug therapy: translational prediction of human brain distribution, target site kinetics, and therapeutic effects. Fluids Barriers CNS. Fluids Barriers CNS. 2013;10(1):1-16.

6. Westerhout J, Danhof M, Lange ECMDE. Preclinical prediction of human brain target site concentrations: considerations in extrapolating to the clinical setting. J Pharm Sci. 2011;100(9):3577-93.

7. Hammarlund-Udenaes M, Fridén M, Syvänen S, Gupta A. On the rate and extent of drug delivery to the brain. Pharm Res. 2008;25(8):1737-50.

8. Abbott NJ. Prediction of blood-brain barrier permeation in drug discovery from in vivo, in vitro and in silico models. Drug Discov Today Technol. 2004;1(4):407-16.

9. de Lange ECM, Hammarlund-Udenaes M. Translational aspects of blood-brain barrier transport and central nervous system effects of drugs: from discovery to patients. Clin Pharmacol Ther. 2015;97(4):380-94.

10. Loryan I, Fridén M, Hammarlund-Udenaes M. The brain slice method for studying drug distribution in the CNS. Fluids Barriers CNS. 2013;10(6):1-9.

11. Loryan I, Sinha V, Mackie C, Van Peer A, Drinkenburg W, Vermeulen A, et al. Mechanistic understanding of brain drug disposition to optimize the selection of potential neurotherapeutics in drug discovery. Pharm Res. 2014;32(8):2203-19.

12. Neuwelt E, Abbott NJ, Abrey L, Banks WA, Blakley B, Davis T, et al. Strategies to advance translational research into brain barriers. Lancet Neurol. 2008;7(1):84-96.

13. Hammarlund-Udenaes M. The use of microdialysis in GNS drug delivery studies: pharmacokinetic perspectives and results with analgesics and antiepileptics. Adv Drug Deliv Rev. 2000;45(2-3):283-94.

14. Van HasseltJGG, Van Der Graaf PH. Towards integrative systems pharmacology models in oncology drug development. Drug Discov Today Technol. 2015;15:1-8. Elsevier Ltd.

15. Collins JM, Dedrick RL. Distributed model for drug delivery to CSF and brain tissue. Am J Physiol. 1983;245(3):R303-10.

16. Liu X, Smith BJ, Chen C, Callegari E, Becker SL, Chen X, et al. Use of a physiologically based pharmacokinetic model to study the time to reach brain equilibrium: an experimental analysis of the role of blood-brain barrier permeability, plasma protein binding, and brain tissue binding. J Pharmacol Exp Ther. 2005;313(3):1254-62.

17. Kielbasa W, Kalvass JC, Stratford R. Microdialysis evaluation of atomoxetine brain penetration and central nervous system pharmacokinetics in rats. Drug Metab Dispos. 2009;37(1):137-42.

18. Kielbasa W, Stratford RE. Exploratory translational modeling approach in drug development to predict human brain pharmacokinetics and pharmacologically relevant clinical doses. Drug Metab Dispos. 2012;40(5):877-83.

19. Badhan RKS, Chenel M, Penny JI. Development of a physiologically-based pharmacokinetic model of the rat central nervous system. Pharmaceutics. 2014;6(1):97-136.

20. Ball K, Bouzom F, Scherrmann J-M, Walther B, Declèves X. Comparing translational population-PBPK modelling of brain microdialysis with bottom-up prediction of brain-to-plasma distribution in rat and human. Biopharm Drug Dispos. 2014;25(8):485-99.

21. Deo AK, Theil F-P, Nicolas J-M. Confounding parameters in preclinical assessment of blood-brain barrier permeation: an overview with emphasis on species differences and effect of disease states. Mol Pharm. 2013;10(5):1581-95.

22. Gaohua L, Neuhoff S, Johnson TN, Rostami-hodjegan A, Jamei M. Development of a permeability-limited model of the human brain and cerebrospinal fluid (CSF) to integrate known physiological and biological knowledge: estimating time varying CSF drug concentrations and their variability using in vitro data. Drug Metab Pharmacokinet. 2016;31(3):224-33.

23. Westerhout J, Ploeger B, Smeets J, Danhof M, de Lange ECM. Physiologically based pharmacokinetic modeling to investigate regional brain distribution kinetics in rats. AAPS J. 2012;14(3):54353.

24. Westerhout J, Smeets J, Meindert D, De Lange ECM. The impact of P-gp functionality on non-steady state relationships between CSF and brain extracellular fluid. J Pharmacokinet Pharmacodyn. 2013;40(3):327-42.

25. Westerhout J, Van Den Berg D-J, Hartman R, Danhof M, De Lange ECM. Prediction of methotrexate CNS distribution in different species - influence of disease conditions. Eur J Pharm Sci. 2014:57:11-24.

26. Stevens J, Ploeger BA, Van Der Graaf PH, Danhof M, De Lange ECM. Systemic and direct nose-to-brain transport pharmacokinetic model for remoxipride after intravenous and intranasal administration. Drug Metab Dispos. 2011;39(12):2275-82.

27. Srikanth CH, Chaira T, Sampathi S, V B S, Bambal RB. Correlation of in vitro and in vivo plasma protein binding using ultracentrifugation and UPLC-tandem mass spectrometry. Analyst. 2013;138(20):6106-16.

28. Widman M, Nilsson L, Bryske B, Lundström J. Disposition of remoxipride in different species. Species differences in metabolism. Arzneimittelforschung. 1993;43(3):287-97.

29. Ståhle L, Segersvärd S, Ungerstedt U. A comparison between three methods for estimation of extracellular concentrations of exogenous and endogenous compounds by microdialysis. J Pharmacol Methods. 1991;25(1):41-25. 
30. Dey M, Jaffe J, Stadnik A, Awad IA. External ventricular drainage for intraventricular hemorrhage. Curr Neurol Neurosci Rep. 2012;12(1):24-33.

31. Bannwarth B, Netter P, Lapicque F, Gillet P, Péré P, Boccard E, et al. Plasma and cerebrospinal fluid concentrations of paracetamol after a single intravenous dose of propacetamol. Br J Clin Pharmacol. 1992;34(1):79-81.

32. Gazzard B, Ford-Hutchinson A, Smith M, Williams R. The binding of paracetamol to plasma proteins of man and pig. J Pharm Pharmacol. 1973;25(12):964-7.

33. Bouw R, Ederoth P, Lundberg J, Ungerstedt U, Nordström C-H, Hammarlund-Udenaes M. Increased blood - brain barrier permeability of morphine in a patient with severe brain lesions as determined by microdialysis Case report. Acta Anaesthesiol Scand. 2001;45(3):390-2.

34. Ederoth P, Tunblad K, Bouw R, Lundberg CJ, Ungerstedt U, Nordstrom $\mathrm{CH}$, et al. Blood-brain barrier transport of morphine in patients with severe brain trauma. Br J Clin Pharmacol. 2003;57(4):427-35.

35. Beal S, Sheiner L, Boeckmann A, Bauer R. NONMEM user's guides. Ellicott City: Icon Development Solutions; 2010.

36. R Core Team. R: A Language and Environment for StatisticalComputing, R Foundation for Statistical Computing. 2013. Available from: http://www.R-project.org/.

37. Sheiner LB, Beal SL. Evaluation of methods for estimating population pharmacokinetics parameters. I. Michaelis-Menten model: routine clinical pharmacokinetic data. J Pharmacokinet Biopharm. 1980;8(6):553-71.

38. Hettiarachchi HDM, Hsu Y, Harris TJ, Penn R, Linninger AA. The effect of pulsatile flow on intrathecal drug delivery in the spinal canal. Ann Biomed Eng. 201 1;39(10):2592-602.

39. Hladky SB, Barrand MA. Mechanisms of fluid movement into, through and out of the brain: evaluation of the evidence. Fluids Barriers CNS. 2014;11(26): 1-32.

40. Skipor J, Thiery JC. The choroid plexus - cerebrospinal fluid system: undervaluated pathway of neuroendocrine signaling into the brain. Acta Neurobiol Exp. 2008;68(3):414-28.

41. Kumar G, Smith QR, Hokari M, Parepally J, Duncan MW. Brain uptake, pharmacokinetics, and tissue distribution in the rat of neurotoxic N-butylbenzenesulfonamide. Toxicol Sci. 2007;97(2):25364 .

42. Strazielle N, Ghersi-Egea JF. Choroid plexus in the central nervous system: biology and physiopathology. J Neuropathol Exp Neurol. 2000;59(7):561-74.

43. Makridakis S. Accuracv concerns measures: theoretical and practical concerns. Int J Forecast. 1993;9:527-9.

44. Begley DJ, Bradbury MW, Kreuter J. The blood-brain barrier and drug delivery to the CNS. New York: Marcel Dekker, Inc.; 2000.

45. Condon P, Wyper D, Grant R, Patterson J, Hadley D, Teasdale G, et al. Use of magnetic resonance imaging to measure intracranial cerebrospinal fluid volume. Lancet. 1986;327(8494):1355-7.

46. Kohn MI, Tanna NK, Herman GT, Resnick SM, Mozley PD, Gur $\mathrm{RE}$, et al. Analysis of brain and cerebrospinal fluid volumes with MR imaging. Part I. Methods, reliability, and validation. Radiology. 1991;178(1):115-22.

47. Dickey CG, Shenton ME, Hirayasu Y, Fischer I, Voglmaier MM, Niznikiewicz MA, et al. Large CSF volume not attributable to ventricular volume in schizotypal personality disorder. Am J Psychiatry. 2000;157(1):48-54.

48. Robertson EG. Developmental defects of the cisterna magna and dura mater. J Neurol Neurosurg Psychiatry. 1949;12(1):39-51.

49. Adam R, Greenberg JO. The mega cisterna magna. J Neurosurg. 1978;48(2):190-2.

50. Pardridge WM. Drug transport in brain via the cerebrospinal fluid. Fluids Barriers CNS. BioMed Cen Ltd. 201 1;8(7):1-7.
51. Mahat MYA, Fakrudeen Ali Ahamed N, Chandrasekaran S, Rajagopal S, Narayanan S, Surendran N, et al. An improved method of transcutaneous cisterna magna puncture for cerebrospinal fluid sampling in rats. J Neurosci Methods. 2012;211(2):272-9. Elsevier BV.

52. Cserrl H. Potassium exchange between cerebrospinal fluid, plasma, and brain. Am J Physiol. 1965;209:1219-26.

53. Cserr H, Cooper D, Suri P, Patlak C. Efflux of radiolabeled polyethylene glycols and albumin from rat brain. Am J Physiol. 1981;240(4):319-28.

54. Abbott NJ. Evidence for bulk flow of brain interstitial fluid: significance for physiology and pathology. Neurochem Int. 2004;45: $545-52$

55. Schinkel AH. P-Glycoprotein, a gatekeeper in the blood-brain barrier. Adv Drug Deliv Rev. 1999;36(2-3):179-94.

56. Tsuji A, Tamai I. Blood-brain barrier function of P-glycoprotein. Adv Drug Deliv Rev. 1997;25(2-3):287-98.

57. Rao VV, Dahlheimer JL, Bardgett ME, Snyder AZ, Finch RA, Sartorelli AC, et al. Choroid plexus epithelial expression of MDR I P glycoprotein and multidrug resistance-associated protein contribute to the blood- cerebrospinal-fluid drug-permeability barrier. Med Sci. 1999;96(7):3900-5.

58. Cordon-Cardo C, O’Brien JP, Casals D, Rittman-Grauer L, Biedler JL, Melamed MR, et al. Multidrug-resistance gene (Pglycoprotein) is expressed by endothelial cells at blood-brain barrier sites. Proc Natl Acad Sci U S A. 1989;86(2):695-8.

59. Kassem NA, Deane R, Segal MB, Chen R, Preston JE. Thyroxine (T4) transfer from CSF to choroid plexus and ventricular brain regions in rabbit: contributory role of P-glycoprotein and organic anion transporting polypeptides. Brain Res. 2007;1 181:44-50.

60. Chen H, Winiwarter S, Fridén M, Antonsson M, Engkvist O. In silico prediction of unbound brain-to-plasma concentration ratio using machine learning algorithms. J Mol Graph Model. 201 1;29(8):985-95. Elsevier Inc.

61. Loryan I, Sinha V, Mackie C, Van Peer A, Drinkenburg WH, Vermeulen A, et al. Molecular properties determining unbound intracellular and extracellular brain exposure of CNS drug candidates. Mol Pharm. 2015;12(2):520-32.

62. Johnson M, Kozielska M, Pilla Reddy V, Vermeulen A, Barton HA, Grimwood S, et al. Translational modeling in schizophrenia: predicting human dopamine D2 receptor occupancy. Pharm Res. 2016;33(4):1003-17.

63. Li CH, Stratford RE, Velez de Mendizabal N, Cremers TI, Pollock $\mathrm{BG}$, Mulsant $\mathrm{BH}$, et al. Prediction of brain clozapine and norclozapine concentrations in humans from a scaled pharmacokinetic model for rat brain and plasma pharmacokinetics. J Transl Med. 2014;12(1):1-11.

64. Melhem M. Perspectives in pharmacology translation of central nervous system occupancy from animal models: application of pharmacokinetic / pharmacodynamic modeling. J Pharmacol Exp Ther. 2013;347:2-6.

65. Stevens J, Ploeger BA, Hammarlund-Udenaes M, Osswald G, Van Der Graaf PH, Danhof M, et al. Mechanism-based PK-PD model for the prolactin biological system response following an acute dopamine inhibition challenge: quantitative extrapolation to humans. J Pharmacokinet Pharmacodyn. 2012;39(5):463-77.

66. Lu Y, Barton HA, Leung L, Zhang L, Hajos-Korcsok E, Nolan CE, et al. Cerebrospinal fluid $\beta$-amyloid turnover in the mouse, dog, monkey and human evaluated by systematic quantitative analyses. Neurodegener Dis. 2013;12(1):36-50.

67. Beaumont A, Marmarou A, Hayasaki K, Barzo P, Fatouros P, Corwin $\mathrm{F}$, et al. The permissive nature of Blood Brain Barrier (BBB) opening in edema formation following traumatic brain injury. Acta Neurochir Suppl. 2000;76:125-9.

68. Bașkaya MK, Rao AM, Doğan A, Donaldson D, Dempsey RJ. The biphasic opening of the blood-brain barrier in the cortex and 
hippocampus after traumatic brain injury in rats. Neurosci Lett. 1997;226(1):33-6.

69. de Lange EC, Danhof M, de Boer AG, Breimer DD. Critical factors of intracerebral microdialysis as a technique to determine the pharmacokinetics of drugs in rat brain. Brain Res. 1994;666(1):1-8.

70. Groenendaal D, Freijer J, de Mik D, Bouw MR, Danhof M, de Lange ECM. Population pharmacokinetic modelling of non-linear brain distribution of morphine: influence of active saturable influx and P-glycoprotein mediated efflux. Br J Pharmacol. 2007;151(5): 701-12.

71. Bouw MR, Gårdmark M, Hammarlund-Udenaes M. Pharmacokinetic-pharmacodynamic modelling of morphine transport across the blood-brain barrier as a cause of the antinociceptive effect delay in rats-a microdialysis study. Pharm Res. 2000;17(10):1220-7.
72. Stevens J, Suidgeest E, van der Graaf PH, Danhof M, de Lange ECM. A new minimal-stress freely-moving rat model for preclinical studies on intranasal administration of GNS drugs. Pharm Res. 2009;26(8):1911-7.

73. Thorne RG, Hrabe S, Nicholson C, Robert G. Diffusion of epidermal growth factor in rat brain extracellular space measured by integrative optical imaging. J Neurophysiol. 2004;92(6):347 1-81.

74. Levinger IM. The cerebral ventricles of the rat.J Anat. 1971;108(3): 447-51.

75. Bass NH, Lundborg P. Postnatal development of bulk flow in the cerebrospinal fluid system of the albino rat: clearance of carboxyl-(14 G)inulin after intrathecal infusion. Brain Res. $1973 ; 52: 323-32$ 